\title{
Continuum viscoplastic simulation of a granular column collapse on large slopes: $\mu(I)$ rheology and lateral wall effects
}

N. Martin, I. R. Ionescu, A. Mangeney, F. Bouchut, and M. Farin

Citation: Phys. Fluids 29, 013301 (2017); doi: 10.1063/1.4971320

View online: http://dx.doi.org/10.1063/1.4971320

View Table of Contents: http://aip.scitation.org/toc/phf/29/1

Published by the American Institute of Physics

\section{Articles you may be interested in}

Modulating flow and aerodynamic characteristics of a square cylinder in crossflow using a rear jet injection Phys. Fluids 29, 015103015103 (2017); 10.1063/1.4972982

Stability analysis of the interface between two weak viscoelastic liquids under periodic oscillations

Phys. Fluids 29, 013101013101 (2017); 10.1063/1.4973515

Splash threshold of a droplet impacting a moving substrate

Phys. Fluids 29, 012103012103 (2017); 10.1063/1.4972976

On the two-dimensional structure of short gravity waves in a wind wave tank

Phys. Fluids 29, 016601016601 (2017); 10.1063/1.4973319 


\title{
Continuum viscoplastic simulation of a granular column collapse on large slopes: $\mu(I)$ rheology and lateral wall effects
}

\author{
N. Martin, ${ }^{1}$ I. R. Ionescu, ${ }^{2,3}$ A. Mangeney, ${ }^{1,4}$ F. Bouchut, ${ }^{5}$ and M. Farin ${ }^{1,6}$ \\ ${ }^{1}$ Institut de Physique du Globe de Paris, Seismology Team, University Paris-Diderot, \\ Sorbonne Paris Cité, France \\ ${ }^{2}$ LSPM, University Paris-Nord, Sorbonne-Paris-Cité, France \\ ${ }^{3}$ IMAR, Romanian Academy, Bucharest, Romania \\ ${ }^{4}$ ANGE Team, CEREMA, INRIA, Laboratoire J. Louis Lions UPMC, Paris, France \\ ${ }^{5}$ Laboratoire d'Analyse et de Mathématiques Appliquées (UMR 8050), CNRS, UPEM, UPEC, \\ Université Paris-Est, 77454 Marne-la-Vallée, France \\ ${ }^{6}$ Seismological Laboratory, California Institute of Technology, Pasadena, California 91125, USA
}

(Received 19 July 2016; accepted 18 November 2016; published online 12 January 2017)

\begin{abstract}
We simulate here dry granular flows resulting from the collapse of granular columns on an inclined channel (up to $22^{\circ}$ ) and compare precisely the results with laboratory experiments. Incompressibility is assumed despite the dilatancy observed in the experiments (up to 10\%). The 2-D model is based on the so-called $\mu(I)$ rheology that induces a Drucker-Prager yield stress and a variable viscosity. A nonlinear Coulomb friction term, representing the friction on the lateral walls of the channel, is added to the model. We demonstrate that this term is crucial to accurately reproduce granular collapses on slopes $\gtrsim 10^{\circ}$, whereas it remains of little effect on the horizontal slope. Quantitative comparison between the experimental and numerical changes with time of the thickness profiles and front velocity makes it possible to strongly constrain the rheology. In particular, we show that the use of a variable or a constant viscosity does not change significantly the results provided that these viscosities are of the same order. However, only a fine tuning of the constant viscosity ( $\eta=1 \mathrm{~Pa} \mathrm{~s}$ ) makes it possible to predict the slow propagation phase observed experimentally at large slopes. Finally, we observed that small-scale instabilities develop when refining the mesh (also called ill-posed behavior, characterized in the work of Barker et al. ["Well-posed and ill-posed behaviour of the $\mu(I)$-rheology for granular flow," J. Fluid Mech. 779, 794-818 (2015)] and in the present work) associated with the mechanical model. The velocity field becomes stratified and the bands of high velocity gradient appear. These model instabilities are not avoided by using variable viscosity models such as the $\mu(I)$ rheology. However we show that the velocity range, the static-flowing transition, and the thickness profiles are almost not affected by them. Published by AIP Publishing. [http://dx.doi.org/10.1063/1.4971320]
\end{abstract}

\section{INTRODUCTION}

Granular materials play a major role in many branches of natural and industrial physics. The description of the mechanical behaviour of these media and the quantification of the rheological parameters involved still remain a challenging question (e.g., work of Bingham, ${ }^{4}$ GDR Midi Group, ${ }^{41}$ Andreotti et al.,$^{2}$ Ancey, ${ }^{1}$ Delannay et al. ${ }^{11}$ ). Detailed quantitative comparison between numerical models and observations is necessary to go further in this direction.

In the context of geophysical flows such as landslides and debris avalanches, most of the numerical work has been performed using thin-layer depth-averaged approximations to overcome the prohibitive computational burden of solving the 3 -D problem. These "shallow" models have proved to be efficient in simulating the final deposit of the flow. However, even though the first order dynamics of the landslides is reproduced, ${ }^{15,33,42,43,53}$ these models fail in describing accurately the flow velocity, especially during the first instants of the mass spreading. In addition, mechanical processes such as the static-flowing transition are not well handled by thin-layer depth-averaged models. ${ }^{5,6}$
As a result, the mechanical investigation of these flows based on a complete 3-D modelling and comparison with laboratory experiments is a relevant way to validate or improve the constitutive relations that are being used. In this context, the experimental and numerical study of the release of rectangular or cylindrical granular columns (so-called granular column collapse) on channelled or unconfined horizontal and inclined beds has become widely used to analyze the dynamics and deposit of gravity granular flows (see, e.g., the work of Delannay et al. ${ }^{11}$ for a review). The reason is that it has a typical and reproducible transient behavior. On horizontal slopes, it starts with a quick acceleration phase that lasts until a maximum velocity is reached. After that, the flow begins to decelerate until it stops. ${ }^{31,34}$ As the slope increases, the duration of the deceleration phase increases. At large slopes $\left(>16^{\circ}\right)$, after the deceleration, a slow propagation phase with a quite stable velocity is observed that increases the duration of the flow and consequently the maximum distance it reaches. ${ }^{13,36}$ The different phases of the flow, the shape of the thickness profiles, and final deposit are the main features that the numerical models try to reproduce. These quantities change with the slope of the bed, the aspect ratio of 
the initial column, the possible presence of an erodible bed, etc.

As a matter of fact, many numerical studies addressed the granular column released problem using typically three different approaches: shallow-type models (Mangeney-Castelnau et al. ${ }^{35}$ Kerswell, ${ }^{27}$ Larrieu et al., ${ }^{32}$ and Doyle et al. ${ }^{12}$ ), Discrete Element Methods (DEM) (Staron and Hinch, ${ }^{51}$ Zenit, ${ }^{54}$ Lacaze et al. ${ }^{29}$ and Girolami et al. $\left.{ }^{16}\right)$, and complete viscousplastic or elasto-plastic models (Crosta et al., ${ }^{8}$ Lacaze and Kerswell, ${ }^{28}$ Meruane et al. ${ }^{40}$ Lagrée et al. ${ }^{30}$ and Ionescu et al. ${ }^{24}$ ).

While shallow models generally well reproduce the experimental final deposit shape after calibration of the friction coefficients, they strongly overestimate the flow velocity during the acceleration phase (see, e.g., the work of Mangeney-Castelnau et al. ${ }^{35}$ Kerswell, ${ }^{27}$ and Ionescu ${ }^{23}$ ). Discrete Element Methods have been able to reproduce more quantitatively the column spreading with well-chosen friction coefficient values (see the work of Lacaze and Kerswell ${ }^{28}$ and Girolami et al. ${ }^{16}$ ) but often overestimate the runout distance observed experimentally (see the work of Staron and Hinch ${ }^{51}$ and Lagrée et al. ${ }^{30}$ ). In addition, all these studies except Ionescu et al. ${ }^{24}$ focus on the collapse of a horizontal plane, and mostly on the reproduction of scaling laws, with little attention paid to the dynamics.

A first attempt to thoroughly compare the flow dynamics simulated with a continuum viscoplastic approach to experimental results (typically by comparing thickness profiles during the collapse) is proposed by Ionescu et al. ${ }^{24}$ Their viscoplastic model includes a Drucker-Prager yield stress and either a variable viscosity (equivalent to the well-known $\mu(I)$ rheology) or a constant viscosity. They accurately predicted the whole spreading of the column on a horizontal plane using values of the rheological parameters taken from the literature, without any calibration process. However, they significantly overestimated the maximum distance reached by the flow for collapses on an inclined plane $\left(\alpha=16^{\circ}\right)$. For the granular collapses they investigated, their simulations with the $\mu(I)$ rheology, i.e., variable viscosity, were very similar to those obtained with a constant viscosity. After this first work, several questions remain: (1) Is the overestimation of the flow dynamics on inclined slopes due to the poor description of lateral wall friction in the model? (2) Is the model able to quantitatively reproduce granular collapses on larger slopes where a slow propagation phase develops? (3) Is the difference between the simulations using the variable viscosity coming from the $\mu(I)$ rheology and the constant viscosity still small for larger slopes? (4) Is the flow stable despite the ill-posed nature of the $\mu(I)$ rheology near the static-flowing transition (Barker et $\left.a .^{3}\right)^{3}$ ?

We address these issues here by using the numerical model proposed in the work of Ionescu et al. ${ }^{24}$ where we added the description of the friction applied by the lateral walls bordering the channel. We mainly focus on the simulation of granular column collapses on moderate slopes (from horizontal to $22^{\circ}$ ) by comparing the results with the laboratory experiments of Mangeney et al. ${ }^{36}$ and Farin et al. ${ }^{13}$

In the first part, we briefly describe the model and how we include lateral friction effects in a 2-D model. In the second part, simulations are compared with experiments. Our results show that the new model makes it possible to reproduce quantitatively column collapses on a large range of slopes, demonstrating the key role of lateral friction, in particular, on the static/flowing interface position. In the third part, comparison between the thickness profiles and front velocities simulated with the variable and constant viscosity is performed for granular collapses on different slopes. Supporting the preliminary results of Ionescu et al. ${ }^{24}$ we demonstrate that fundamental changes in the flow dynamics in our case can be obtained when changing the average value of the viscosity while its spatio-temporal evolution during the flow poorly affects the results. Pressure profiles are compared to analytical profiles derived from a constant viscosity Drucker-Prager model to highlight the similarities between the results obtained with constant and variable viscosities. On the fourth part, we present simulations showing the development of unstable shear bands when one refines the mesh. Finally, we discuss the implication of our results in terms of rheological modeling and numerical simulations.

\section{GRANULAR MATERIAL MECHANICAL MODEL}

A granular material generally exhibits a plastic behavior that prevents the medium from deforming if the applied stress is lower than a given value called yield stress. This effect is modeled using a plasticity criterion that describes this flow/noflow behavior. In the specific case of a granular material, the plasticity criterion is a frictional criterion in the sense that its ability to sustain a Cauchy stress $\sigma$ is due to internal friction between grains and geometrical particle arrangement.

A classical plasticity criterion that describes the flow/noflow condition is the Drucker-Prager plasticity criterion,

$$
\|\boldsymbol{S}\| \leq \kappa(p)=\kappa_{0}+\mu p,
$$

if and only if the fluid is at rest. Here $\|\boldsymbol{A}\|=\sqrt{\boldsymbol{A}: \boldsymbol{A} / 2}$ is the Frobenius matrix norm (the second invariant), $S$ is the deviatoric stress tensor $(\sigma=-p \mathrm{Id}+\boldsymbol{S}), \kappa$ is the von Mises yield limit, $p$ is the pressure, $\mu=\tan (\delta)$ is the tangent of the internal friction angle, and $\kappa_{0}$ is the cohesion of the material, set to 0 here.

With a von Mises plasticity criterion, $\kappa(p) \equiv \kappa^{0}>0, \mu=0$ and a constant viscosity, one can recover the classical Bingham fluid model (see Ref. 4) used for many fluids with a solid like behavior (for instance, oils or sediments in oil drilling processes). This model and the regularized one, usually called Bingham-Papanastasiou model (see Ref. 44), were also considered to describe the (high rate) deformation of many solid materials having a fluid like behavior. However, these models are not able to paint the dependency of the yield limit with pressure specific to granular materials (see, e.g., the work of Andreotti et ll $^{2}$ for a review of the behaviour of granular materials).

The deformation of the material occurring under a large enough stress is given by a visco-plastic flow law that makes the hypothesis of the collinearity of the deviatoric stress tensor $\boldsymbol{S}$ and the strain-rate tensor $\boldsymbol{D}=\frac{1}{2}\left(\nabla \mathbf{u}+\nabla \mathbf{u}^{T}\right)$, where $\mathbf{u}$ denotes the velocity field. By analogy to a viscous flow law, in the $\mu(I)$ rheology, 


$$
\boldsymbol{S}=\mu(I) p \frac{\boldsymbol{D}}{\|\boldsymbol{D}\|},
$$

the term $\mu p /\|\boldsymbol{D}\|$ can be seen as an effective viscosity of the material depending on shear rate and pressure. This constitutive law was introduced by Jop et al. ${ }^{25}$ to phenomenologically describe the spatio-temporal variability of the internal friction coefficient $\mu$ through a function of one dimensionless quantity called the inertial number $I$. The inertial number, which is the square root of the Savage number or of the Coulomb number, introduced by Savage ${ }^{47}$ and Ancey et al., ${ }^{1}$ respectively, is defined by

$$
I=\frac{2\|\boldsymbol{D}\| d}{\sqrt{p / \rho_{s}}},
$$

where $d$ denotes the grain diameter and $\rho_{s}$ the grain density. This number can be seen as the ratio between two time scales: the microscopic time scale of particle rearrangement $d / \sqrt{p / \rho_{s}}$ and the macroscopic strain rate time scale $1 /\|D\|$. The proportionality factor $\mu(I)$ is then written as

$$
\mu(I)=\mu_{1}+\frac{\mu_{2}-\mu_{1}}{1+\frac{I_{0}}{I}},
$$

where $I_{0}$ is a dimensionless constant, and $\mu_{1}$ and $\mu_{2}$ represent the value of the internal friction coefficient for low and high inertial numbers, respectively.

As it follows from the work of Ionescu et al., ${ }^{24}$ using (4) and (3) in the visco-plastic law (2) leads to

$$
\boldsymbol{S}=\mu_{1} p \frac{\boldsymbol{D}}{\|\boldsymbol{D}\|}+2 \frac{\left(\mu_{2}-\mu_{1}\right) p}{2\|\boldsymbol{D}\|+I_{0} \sqrt{p} / k} \boldsymbol{D},
$$

where $k=d \sqrt{\rho_{s}}$. In that form, the flow law is now including the original pressure-dependent plastic (rate independent) term $\mu_{1} p \boldsymbol{D} /\|\boldsymbol{D}\|$ plus an additional viscous (rate dependent) term $2 \eta_{I} \boldsymbol{D}$ introducing a spatio-temporally varying viscosity depending on the pressure and the shear-rate given by

$$
\eta_{I}=\eta_{I}(p,\|\boldsymbol{D}\|)=\frac{\left(\mu_{2}-\mu_{1}\right) p}{2\|\boldsymbol{D}\|+I_{0} \sqrt{p} / k} .
$$

To sum-up, the rewriting of the $\mu(I)$ rheology naturally leads to a model including the Drucker-Prager plasticity and a variable viscosity which does not directly depend on the inertial number $I$ but on the physical parameters that are involved in the calculation of $I$,

$$
\boldsymbol{S}=\mu(I) p \frac{\boldsymbol{D}}{\|\boldsymbol{D}\|}=\mu_{1} p \frac{\boldsymbol{D}}{\|\boldsymbol{D}\|}+2 \eta_{I} \boldsymbol{D} .
$$

In what follows, the role of the spatio-temporal variation of the viscosity $\eta=\eta_{I}$ is being assessed and compared to the model with a constant viscosity $\eta=\eta_{c}$,

$$
\boldsymbol{S}=\mu_{1} p \frac{\boldsymbol{D}}{\|\boldsymbol{D}\|}+2 \eta_{c} \boldsymbol{D} .
$$

To avoid confusion, the $\mu(I)$ rheology will designate the original definition given by (4) with a variable viscosity $\eta=\eta_{I}$ defined by (6). The model (8) with a constant viscosity $\eta=\eta_{c}$ will be called the Drucker-Prager model.

\section{MASS AND MOMENTUM CONSERVATION}

To include the three dimensional effect of friction on the lateral walls of the channel, let us consider the 3-D

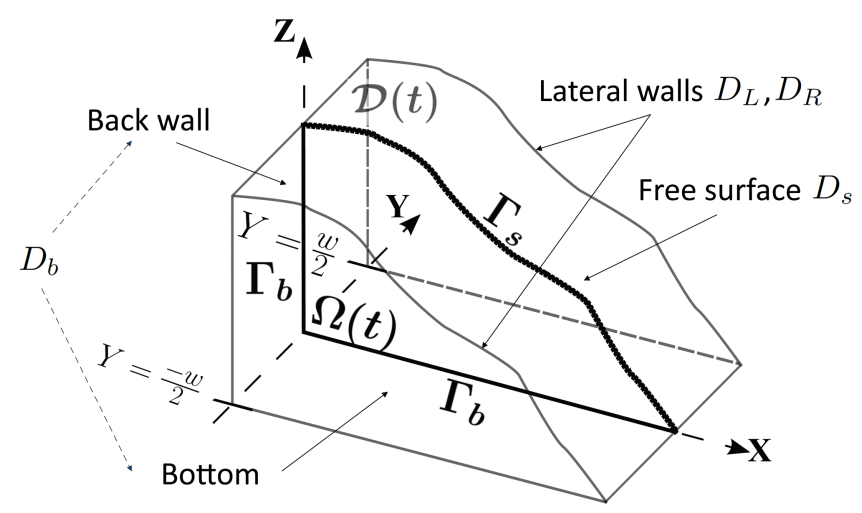

FIG. 1. Fluid domain and notations.

domain $\mathcal{D}(t)=\Omega(t) \times[-w / 2, w / 2]$, where $w$ denotes the channel width, and the bi-dimensional fluid domain $\Omega(t)$ represents the central vertical cross section of the granular flow in the channel (see Figure 1$)$. In what follows, $(x, z ; y)$ denotes the Cartesian (gravity-related) frame (see Figure 2), whereas ( $X, Z$; $Y$ ) denotes the topography (channel-related) frame (see Figure 1) with naturally $Y=y$.

The release from rest of a granular column on a plane or in a channel is a highly transient problem with an aspect ratio of the granular mass close to 1 at the beginning of the collapse. For these reasons, it is modeled here by a complete viscoplastic problem with free-surface transport. The visco-plastic fluid is considered to be incompressible. The solution $(\boldsymbol{u}, p)$ of
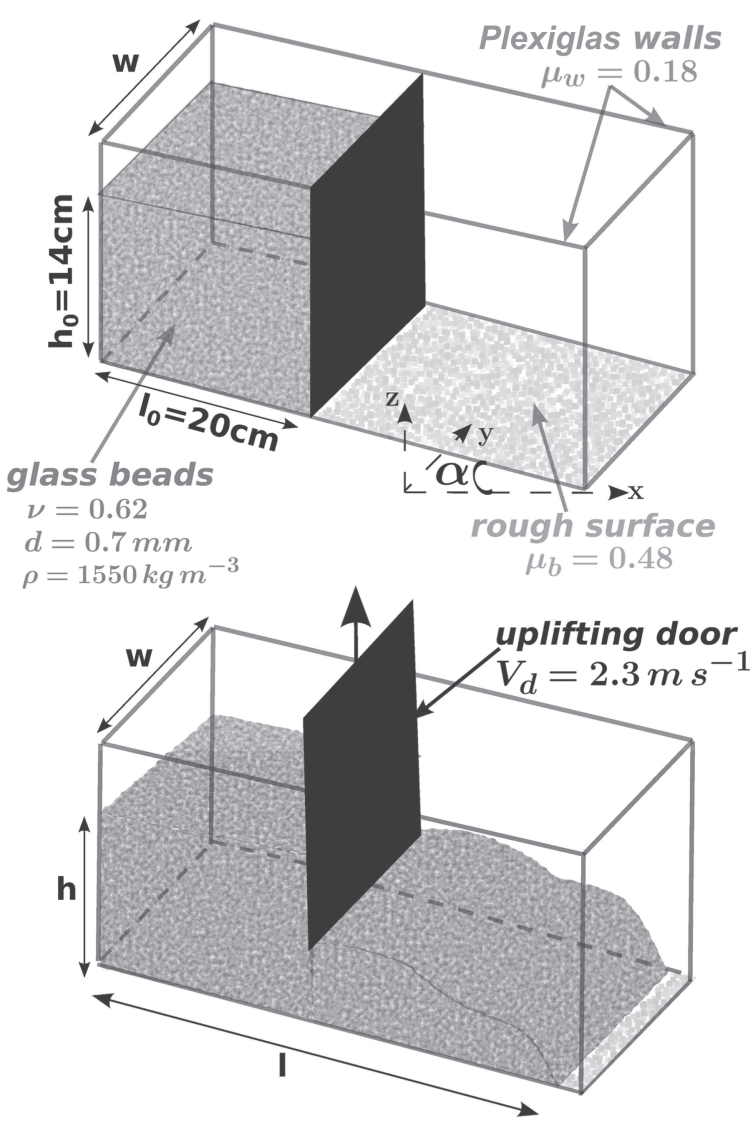

FIG. 2. Sketch of the experimental setup. 
the momentum balance law is computed in the domain $\mathcal{D}(t)$,

$$
\left\{\begin{array}{l}
\rho\left(\partial_{t} \boldsymbol{u}+(\boldsymbol{u} \cdot \nabla) \boldsymbol{u}\right)+\nabla p-\operatorname{div}(\boldsymbol{S})=\rho \boldsymbol{g} \\
\operatorname{div}(\boldsymbol{u})=0
\end{array}\right.
$$
fluid as

The viscoplastic fluid domain $\mathcal{D}(t)$ is transported with the

$$
\frac{\partial 1_{\mathcal{D}(t)}}{\partial t}+\boldsymbol{u} \cdot \nabla 1_{\mathcal{D}(t)}=0
$$

where $1_{\mathcal{D}(t)}$ is the characteristic function of the domain.

\section{BOUNDARY CONDITIONS}

Following the decomposition of the boundary $\partial \Omega(t)$ into two disjoints parts $\partial \Omega(t)=\Gamma_{b}(t) \cup \Gamma_{s}(t)$, we will decompose the boundary of $\mathcal{D}(t)$ into four parts $\partial \mathcal{D}(t)=D_{L}(t)$ $\cup D_{R}(t) \cup D_{b}(t) \cup D_{s}(t): 2$ lateral boundaries $D_{L}(t)=\Omega(t)$ $\times\{-w / 2\}, D_{R}(t)=\Omega(t) \times\{w / 2\}$, the bottom and left wall lateral boundary $D_{b}(t)=\Gamma_{b}(t) \times(-w / 2, w / 2)$, and the free surface $D_{s}(t)=\Gamma_{s}(t) \times(-w / 2, w / 2)$ (Figure 1).

We adopt the following notation for the normal and tangential components of the velocity $\boldsymbol{u}$ and the stress vector $\sigma n$ :

$$
\boldsymbol{u}=u_{n} \boldsymbol{n}+\boldsymbol{u}_{\boldsymbol{T}} \text { and } \boldsymbol{\sigma} \boldsymbol{n}=\sigma_{n} \boldsymbol{n}+\boldsymbol{\sigma}_{\boldsymbol{T}},
$$

where $\boldsymbol{n}$ is the outward normal vector on $\partial \mathcal{D}(t)$.

On the boundaries $D_{b}, D_{R}$ and $D_{L}$, the flowing material is in a frictional contact with a rigid body:

$$
\boldsymbol{u} \cdot \boldsymbol{n}=0, \quad \boldsymbol{\sigma}_{T}=\boldsymbol{F}^{f},
$$

where $\boldsymbol{F}^{f}$ is the frictional stress acting on that boundary and described by the Coulomb friction law,

$$
\left\{\begin{array}{l}
\boldsymbol{F}^{f}=-\mu^{f}\left[-\sigma_{n}\right]_{+} \frac{\boldsymbol{u}_{T}}{\left|\boldsymbol{u}_{T}\right|} \text { if } \boldsymbol{u}_{T} \neq 0 \\
\left|\boldsymbol{F}^{f}\right| \leq \mu^{f}\left[-\sigma_{n}\right]_{+} \text {if } \boldsymbol{u}_{T}=0
\end{array}\right.
$$

Here $\mu^{f}$ is the Coulomb friction coefficient at the boundary, and []$_{+}$is the positive part $\left([s]_{+}=(s+|s|) / 2\right)$. Note that the boundary friction coefficient $\mu^{f}$ is generally different from the internal friction coefficient $\mu_{1}$ and it is a specific data of each contact surface. We consider here two different values for the (boundary) Coulomb friction coefficient $\mu^{f}$,

$\mu^{f}=\left\{\begin{array}{l}\mu_{w}^{f} \text { on the back wall and on the lateral walls, } \\ \mu_{b}^{f} \text { on the rough bottom. }\end{array}\right.$

At the free surface, a stress-free condition is imposed,

$$
\boldsymbol{\sigma n}=0, \quad \text { on } D_{s}(t)
$$

\section{ACCOUNTING FOR LATERAL FRICTION}

Our approach consists in introducing the frictional stresses associated with the two lateral walls $D_{L}$ and $D_{R}$, through a 3-D variational formulation but keeping the in-plane flow assumption to avoid 3-D computations.
The variational formulation of problem (9) can be written as

$$
\begin{aligned}
& \int_{-\frac{w}{2}}^{\frac{w}{2}} \int_{\Omega(t)}\left(\rho\left(\partial_{t} \boldsymbol{u}+\boldsymbol{u} \cdot \nabla \boldsymbol{u}-\boldsymbol{g}\right) \cdot \boldsymbol{\varphi}+\boldsymbol{\sigma}: \boldsymbol{D}(\boldsymbol{\varphi})\right) d x \\
& \quad=\int_{\partial \mathcal{D}(t)} \boldsymbol{\sigma n} \cdot \boldsymbol{\varphi} d x
\end{aligned}
$$

where $\partial \mathcal{D}(t)=D_{L}(t) \cup D_{R}(t) \cup D_{b}(t) \cup D_{s}(t)$ (using notations defined in Sec. IV) is the boundary of the $3 \mathrm{D}$ domain $\mathcal{D}(t)$ and $\varphi$ is a kinematically admissible test function $(\boldsymbol{\varphi} \cdot \boldsymbol{n}=0$ on $\left.D_{L}(t) \cup D_{R}(t) \cup D_{b}(t)\right)$. Using the stress-free condition (15) and the frictional condition (12), we get

$$
\int_{\partial \mathcal{D}(t)} \boldsymbol{\sigma} \boldsymbol{n} \cdot \varphi d x=\int_{D_{L} \cup D_{R}} \boldsymbol{F}_{w}^{f} \cdot \boldsymbol{\varphi}_{T} d s+\int_{D_{b}} \boldsymbol{F}_{b}^{f} \cdot \boldsymbol{\varphi}_{T} d x
$$

where $\boldsymbol{F}_{w}^{f}$ is the friction force acting on $D_{L}(t) \cup D_{R}(t)$ while $\boldsymbol{F}_{b}^{f}$ is acting on $D_{b}(t)$.

Except the lateral friction, already taken into consideration, we will adopt here the in-plane flow assumption. In particular, we shall neglect the variation of the velocity along the transverse direction $Y$ even though experimental results show smaller velocities near the lateral walls than near the center of the flow. In what follows, all the mechanical variables will be assumed to be constant in the transverse direction $Y=y$ (see Figure 1). Bearing in mind that $u_{y}$ is vanishing on $D_{L}(t) \cup D_{R}(t)$, we deduce that there is no transverse flow $u_{y}$ $\equiv 0$, i.e., $\boldsymbol{u}=\left(u_{x}, u_{z}, 0\right)$. We shall use the notation $\boldsymbol{u}=\left(u_{x}, u_{z}\right)$ for the in-plane flow also. Following this assumption, the strain rate and the stress deviator are plane tensors, and we will use the same notations, $\boldsymbol{D}(\boldsymbol{u})$ and $\boldsymbol{S}$, respectively, as for the 3-D description.

On the lateral faces $D_{L}(t) \cup D_{R}(t)$, the normal stress is then given by $\sigma_{n}=\sigma_{y y}=-p$ while the 3-D tangential slip rate is the in-plane velocity, i.e., $\boldsymbol{u}_{T}=\boldsymbol{u}$. The frictional stress acting on the lateral walls is

$$
\left\{\begin{array}{l}
\boldsymbol{F}_{w}^{f}=-\mu_{w}^{f}[p]_{+} \frac{\boldsymbol{u}}{|\boldsymbol{u}|} \text { if } \boldsymbol{u} \neq 0, \\
\left|\boldsymbol{F}_{w}^{f}\right| \leq \mu_{w}^{f}[p]_{+} \text {if } \boldsymbol{u}=0,
\end{array}\right.
$$

while the frictional stress acting on the bottom and on the left side is

$$
\left\{\begin{array}{l}
\boldsymbol{F}_{b}^{f}=-\mu^{f}\left[-\sigma_{n}\right]_{+} \frac{\boldsymbol{u}_{T}}{\left|\boldsymbol{u}_{T}\right|} \text { if } \boldsymbol{u}_{T} \neq 0 \\
\left|\boldsymbol{F}_{b}^{f}\right| \leq \mu^{f}\left[-\sigma_{n}\right]_{+} \text {if } \boldsymbol{u}_{T}=0
\end{array}\right.
$$

where $\mu^{f}=\mu_{w}^{f}$ on the back wall and $\mu^{f}=\mu_{b}^{f}$ on the rough bottom. Then, the boundary term (16) becomes

$$
\int_{\partial \mathcal{D}(t)} \boldsymbol{\sigma} \boldsymbol{n} \cdot \boldsymbol{\varphi} d x=w \int_{\Gamma_{b}} \boldsymbol{F}_{b}^{f} \cdot \boldsymbol{\varphi}_{T} d s+2 \int_{\Omega(t)} \boldsymbol{F}_{b}^{f} \cdot \boldsymbol{\varphi} d x
$$

The solution being constant in the direction $y$, we can divide now the variational formulation by the width $w$, and we finally obtain

$$
\begin{aligned}
& \int_{\Omega(t)}\left(\rho\left(\partial_{t} \boldsymbol{u}+\boldsymbol{u} \cdot \nabla \boldsymbol{u}-\boldsymbol{g}\right) \cdot \boldsymbol{\varphi}-p \operatorname{div}(\boldsymbol{\varphi})\right) d x \\
& \quad=\int_{\Omega(t)}\left(-\boldsymbol{S}: \boldsymbol{D}(\boldsymbol{\varphi})+\frac{2}{w} \boldsymbol{F}_{w}^{f} \cdot \boldsymbol{\varphi}\right) d x+\int_{\Gamma_{b}} \boldsymbol{F}_{b}^{f} \cdot \boldsymbol{\varphi}_{T} d s,
\end{aligned}
$$


which can be written as an in-plane momentum balance law in $\Omega(t)$,

$$
\rho\left(\partial_{t} \boldsymbol{u}+(\boldsymbol{u} \cdot \nabla) \boldsymbol{u}\right)+\nabla p-\operatorname{div}(\boldsymbol{S})=\frac{2}{w} \boldsymbol{F}_{w}^{f}+\rho \boldsymbol{g} .
$$

It corresponds to the original 2-D momentum balance law from the work of Ionescu et al. $^{24}$ plus the 2-D Coulomb friction term $\frac{2}{w} \boldsymbol{F}_{w}^{f}$. Note that this term which is of order of

$$
\frac{2}{w} \mu_{w}^{f} p \frac{\boldsymbol{u}}{|\boldsymbol{u}|}
$$

induces a frictional resistance in the opposite direction to the velocity.

Ionescu et al. ${ }^{24}$ used another approach to take into account the effect of lateral friction. This consists in increasing the internal friction coefficient by adding to it a constant value of the order of $\mu_{w}^{f} \frac{h}{w}$, where $h$ is the averaged thickness of the flowing layer observed in the experiments (see also Equation (1) of Taberlet et al..$^{52}$ or Equation (4.5) of Jop et al. ${ }^{26}$ ). Indeed, if one replaces $\mu_{1}$ and $\mu_{2}$ by $\left(\mu_{1}+\mu_{w}^{f} \frac{h}{w}\right)$ and $\left(\mu_{2}+\mu_{w}^{f} \frac{h}{w}\right)$, respectively, in Equation (5), then the viscous term remains identical (since it is a function of $\left(\mu_{2}-\mu_{1}\right)$ ). It introduces the following contribution in the momentum equation:

$$
\frac{h}{w} \mu_{w}^{f} \operatorname{div}\left(p \frac{\boldsymbol{D}}{\|\boldsymbol{D}\|}\right) .
$$

For a laminar shear flow $\left(u_{x}=V y / h, u_{y}=0\right)$ with a hydrostatic pressure distribution $p=\rho g(h-y) \cos (\alpha)$, this last term is $\left(\frac{h}{w} \mu_{w}^{f} \rho g \cos (\alpha), 0\right)$ which represents exactly the depth average of the wall frictional force given by Equation (20). However, this similarity is present only for a laminar flow and it is not anymore valid for a non-laminar flow considered here. In the case of (21), the extra friction develops in the direction of the divergence of the strain-rate. In the present case, the term (20) acts in the opposite direction of the velocity $\boldsymbol{u}$. It is important to point out that our approach leads to a non-linear correction that depends on the unknown velocity whereas the approach of Ionescu et al. ${ }^{24}$ linearly modifies the problem by just increasing the internal friction coefficient. This additional constant internal friction consequently slows down the motion (decreases the velocity $\boldsymbol{u}$ ) by changing the position of the static-flowing interface but without qualitatively modifying the velocity field (the dynamics is preserved). On the contrary, the extra friction introduced by (20) adds a contribution to the velocity field that non-linearly counteracts the flow and modifies the shape of the static-flowing interface and of the velocity field (see Section VII). It is thus active whenever a motion occurs.

Details of the numerical algorithm that implements the solution to the problem with lateral friction effect are given in Appendix A. In the following numerical results, meshes are made of 1000 to 2000 triangular cells depending on the situation (except specified otherwise), for an average size of $0.006 \mathrm{~m}$. Times of computation are strongly depending on the rate of convergence which is related to the slope, the frictional parameters, and the numerical parameters among others. Overall, the solution of one time step takes place between a few minutes at the beginning of the collapse, when many $(\sim 1500)$ iterations are needed to achieve the convergence, to a few seconds, later in the simulation, when the initial guess is close
TABLE I. Rheological parameters.

\begin{tabular}{cccccc}
\hline \hline$\mu_{1}$ & $\mu_{2}$ & $\mu_{b}$ & $\mu_{w}$ & $I_{0}$ & $k$ \\
\hline 0.48 & 0.73 & 0.48 & 0.18 & 0.279 & $0.035 \mathrm{~kg}^{1 / 2} \mathrm{~m}^{-1 / 2}$ \\
\hline \hline
\end{tabular}

to the converged solution. The time step is constant in what follows and equal to $0.001 \mathrm{~s}$ thus leading to simulations made of 1500 to 3500 time steps, depending on the slope. Note that, in the studied configurations, the computation with the variable viscosity $\eta_{I}$ induces a higher computational burden and a slower convergence that can lead to overall simulations twice as long as those with a constant viscosity.

\section{EXPERIMENTAL SETUP AND MODEL PARAMETERS}

Let us recall here the experimental setup of Mangeney et al. ${ }^{36}$ and Farin et al. ${ }^{13}$ that is being reproduced numerically in the present work (see also the work of Ionescu et al. ${ }^{24}$ ).

It consists of a channel of rectangular section with Plexiglas walls and a variable spacing $w(w=10 \mathrm{~cm}$ and $w=20 \mathrm{~cm}$ are considered hereafter). A mass of glass beads of height $h_{0}$ $=14 \mathrm{~cm}$ and of length $l_{0}=20 \mathrm{~cm}$ is released from a reservoir at time $t=0 \mathrm{~s}$ by lifting a gate. The glass beads are subspherical, cohesionless, and highly rigid with a diameter $d=0.7 \pm 0.1 \mathrm{~mm}$. They flow down an inclined surface of inclination $\alpha$ roughened with one glued layer of the same glass beads (see Figure 2). The particle density is $\rho_{s}=2500 \mathrm{~kg}$ $\mathrm{m}^{-3}$ and the volume fraction is estimated at $v=0.62$ giving an apparent density $\rho=1550 \mathrm{~kg} \mathrm{~m}^{-3}$. The thickness profiles of the granular mass are being recorded at various instants during the flow.

In the model, the gate is simulated as a rigid boundary with a free-slip condition that is lifted at a velocity $V_{d}=2.3 \mathrm{~m} \mathrm{~s}^{-1}$ (see the work of Ionescu et al. ${ }^{24}$ for details on the effect of the gate on the flow). The various rheological parameters are identical to those used in the work of Ionescu et al. ${ }^{24}$ and are summarized in Table I. At the bed and along the back wall of the reservoir, $\mu=\mu_{b}$, while on the lateral walls, $\mu=\mu_{w}$ (see Figure 2).

\section{LATERAL WALL EFFECTS}

This section presents the results computed from the numerical model described here before for different angles of the channel ranging from $\alpha=0^{\circ}$ to $\alpha=22^{\circ}$. A specific attention is paid to the improvement provided by the introduction of lateral friction effects, particularly at large slopes (typically $\alpha \geq 16^{\circ}$ ) where, as already mentioned, their absence has been thought to be a strong limitation (see the work of Ionescu et al. $^{24}$ ). In the present formulation, the case without lateral friction effects, i.e., $w=\infty$, is simulated using a width $w$ $=10^{10} \mathrm{~m}$ (see Equation (19)).

We simulate here some experiments of Mangeney et al. ${ }^{36}$ and Farin $e t a l .{ }^{13}$ that differ only by the width of the channel, i.e., $w=10 \mathrm{~cm}$ and $w=20 \mathrm{~cm}$, respectively. For both experiments, the initial granular column is rectangular, $20 \mathrm{~cm}$ long and $14 \mathrm{~cm}$ high (see Figure 2) leading to volumes of $2800 \mathrm{~cm}^{3}$ and $5600 \mathrm{~cm}^{3}$, respectively. 
First, we compare the observed and computed thickness profiles of the granular material for different slopes $\alpha\left(10^{\circ}\right.$, $22^{\circ}$ ) for the two different channel widths (Figures 3 and 4). On these figures, the latest plotted instant corresponds to the time when the whole mass has stopped in the experiments. An estimate of the position of the static-flowing interface predicted by the model is obtained by representing the isocontour of a velocity equal to $0.01 \mathrm{~m} \mathrm{~s}^{-1}$. This chosen small velocity corresponds to approximately $1 \%$ of the mean flow velocity during the collapse. The thickness and static-flowing interface profiles computed without lateral friction, corresponding to the results presented in the work of Ionescu et al. ${ }^{24}$ are plotted on every figure to highlight the (positive) effects of the lateral friction term.

Before going into the details of the comparison, a first general observation is that including the lateral friction term (see Equation (19)) systematically improves the results of the simulations. Furthermore, this effect increases as the slope angle increases. It is really significant at large slopes (see, e.g., the results at $22^{\circ}$ in Figure 4). This makes sense since the effect of the Coulomb friction arises only in the flowing zone and this flowing zone represents a larger part of the whole fluid domain when the slope increases. This observation supports the assumption made by Ionescu et al. ${ }^{24}$ that the absence of lateral wall friction in their model explained the deteriorating agreement they found with experimental results as the slope increases.

Another global observation on the shape of the computed thickness profiles is the presence, particularly for $\alpha \geq 16^{\circ}$, of a slight yet systematic lowering of the surface at the upper-left corner. This shape is a numerical artifact related to the remeshing method and disappears for more refined meshes. Note that the scale on the vertical axis is exaggerated, thus enhancing the horizontal variations of the free surface shape.

\section{A. Final deposits}

The computed final shape and runout distance of the deposits are significantly improved by including lateral friction. The average surface slope of the deposit computed with lateral friction is greater than without lateral friction. The concavities and convexities of the surface are more pronounced leading to a better agreement with the experimental profiles.

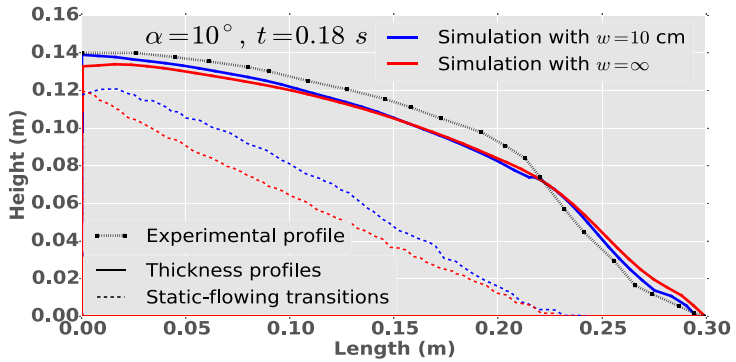

(a) $w=10 \mathrm{~cm}, t=0.18 \mathrm{~s}$

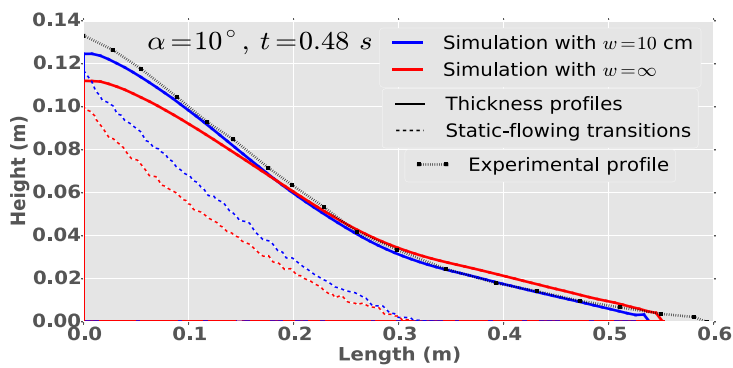

(c) $w=10 \mathrm{~cm}, t=0.48 \mathrm{~s}$

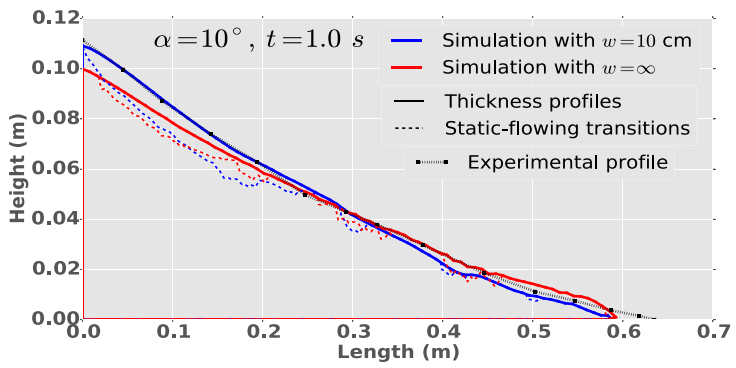

(e) $w=10 \mathrm{~cm}, t=1.0 \mathrm{~s}$

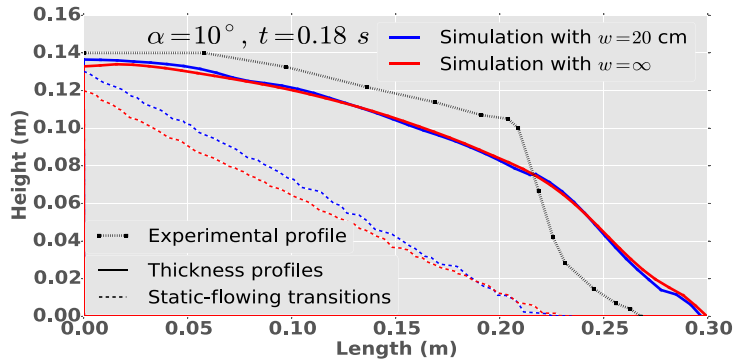

(b) $w=20 \mathrm{~cm}, t=0.18 \mathrm{~s}$

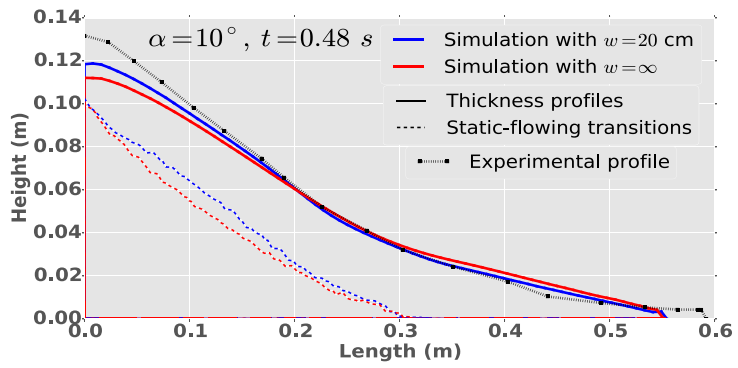

(d) $w=20 \mathrm{~cm}, t=0.48 \mathrm{~s}$

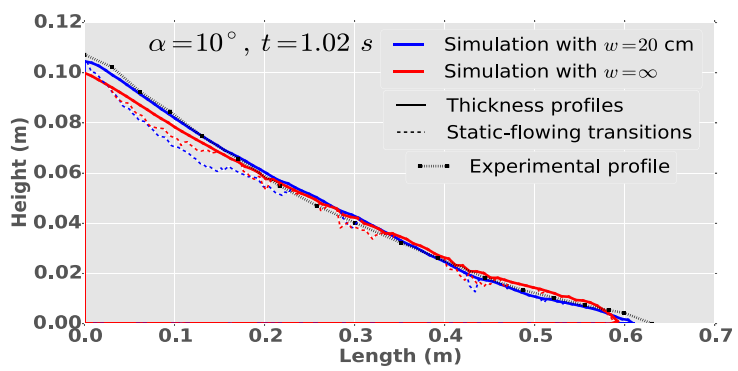

(f) $w=20 \mathrm{~cm}, t=1.02 \mathrm{~s}$

FIG. 3. Computed and experimental thickness profiles for an inclination of the channel $\alpha=10^{\circ}$ computed with the $\mu(I)$ rheology with and without lateral friction effects for $w=10 \mathrm{~cm}$ (left column) and $w=20 \mathrm{~cm}$ (right column) and corresponding computed static-flowing transitions. The axis scales are different for each time and the aspect ratio is thus not preserved. 


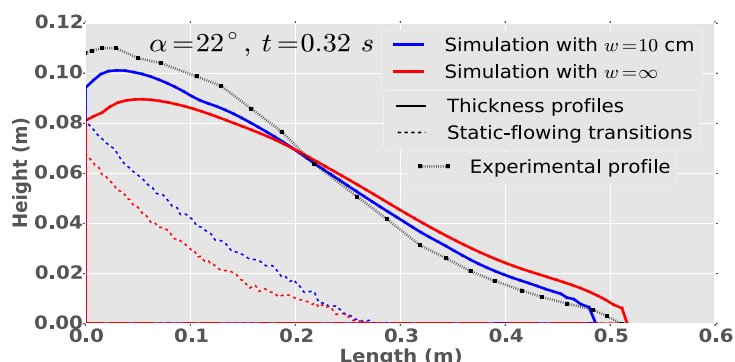

(a) $w=10 \mathrm{~cm}, t=0.32 \mathrm{~s}$

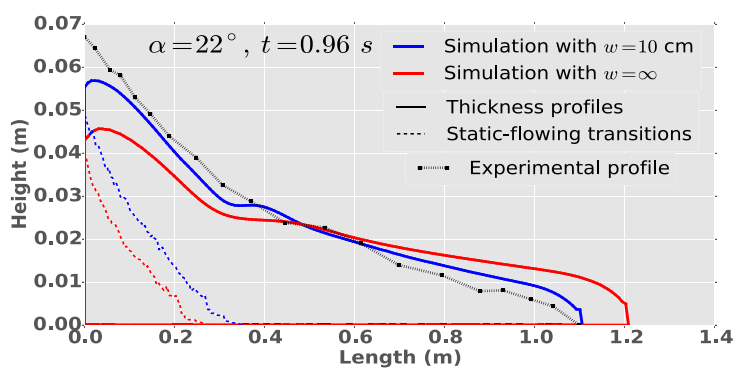

(c) $w=10 \mathrm{~cm}, t=0.96 \mathrm{~s}$

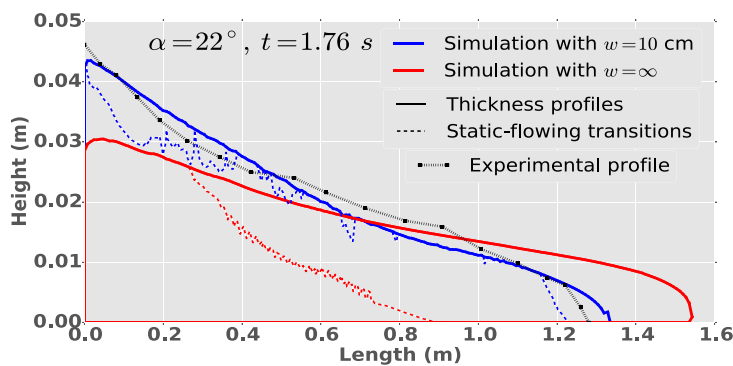

(e) $w=10 \mathrm{~cm}, t=1.76 \mathrm{~s}$

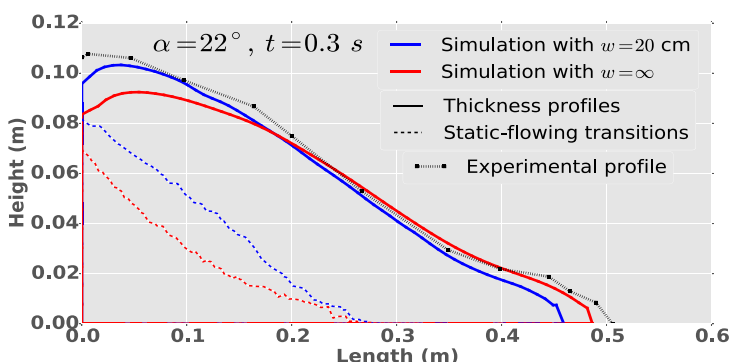

(b) $w=20 \mathrm{~cm}, t=0.3 \mathrm{~s}$

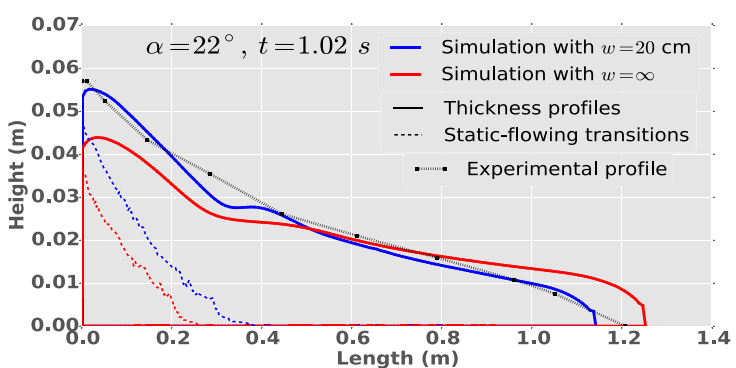

(d) $w=20 \mathrm{~cm}, t=1.02 \mathrm{~s}$

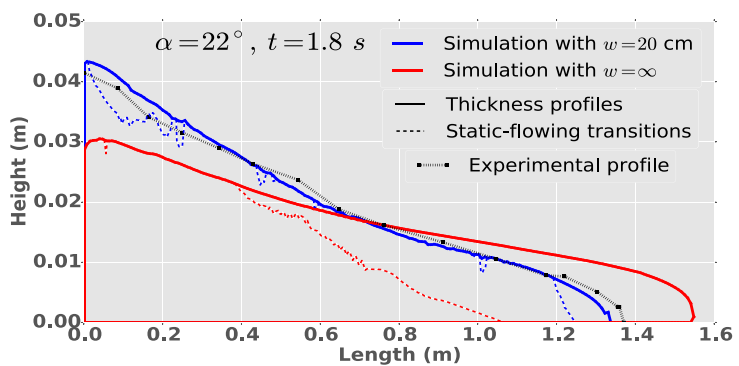

(f) $w=20 \mathrm{~cm}, t=1.8 \mathrm{~s}$

FIG. 4. Computed and experimental thickness profiles for an inclination of the channel $\alpha=22^{\circ}$ computed with the $\mu(I)$ rheology with and without lateral friction effects for $w=10 \mathrm{~cm}$ (left column) and $w=20 \mathrm{~cm}$ (right column) and corresponding computed static-flowing transitions. The axis scales are different for each time and the aspect ratio is thus not preserved.

This observation holds regardless of the width of the channel. Even though the lateral frictional effects are smaller for $w$ $=20 \mathrm{~cm}$ than for $w=10 \mathrm{~cm}$, they remain clearly observable and significantly improve the agreement with experimental results. Note that on a smaller slope $\alpha=10^{\circ}$, the improvement of the results thanks to lateral friction effects is very small.

For $\alpha=10^{\circ}$ (Figures 3(e) and 3(f)), the static-flowing transitions at the time the experimental mass has stopped show that most of the numerical mass has clearly stopped and only very small areas of non-zero velocity close to the surface remain. This motion is too small to lead to any observable mass transfer. The extents of the deposits are very accurate for both cases $w=10 \mathrm{~cm}$ and $w=20 \mathrm{~cm}$. Lateral friction term provided a small yet clear improvement of the computed shape of the surface (obviously more significant for $w=10 \mathrm{~cm}$ ).

For $\alpha=22^{\circ}$ (Figures 4(e) and 4(f)), both the extent and shape of the deposits are significantly improved with the lateral friction term. Indeed, the simulated deposit is very close to the observed one. In particular, the lateral friction term corrects the overestimation of the runout distance computed without lateral friction. At $\alpha=22^{\circ}$ and whatever $w$, the position of the static-flowing transition in the simulations with lateral friction shows that there is still some velocity, in particular near the front. However, this motion is again too small to produce any significant extra propagation (smaller than $0.5 \%$ of the plotted runout distance). On the contrary, simulations without lateral friction show a large still flowing area that will continue to transfer mass downstream and lead to a computed runout distance reaching $1.6 \mathrm{~m}$, much larger than the observed runout distance.

\section{B. Dynamics}

\section{Thickness profiles and static-flowing transition}

The lateral friction term changes the position of the staticflowing transition during the flow. In every case, the lateral friction leads to an interface with a smaller curvature and the angle between the static-flowing interface and the bottom of the channel is also greater. The part of the domain where the mass is flowing is thus reduced. This prevents in particular the downstream and the down flow in the direction normal to the bed of the mass from the upper-left corner (see, e.g., Figure 19 in the work of Ionescu et al. for the vertical velocities in the collapse on a horizontal plane simulated without lateral friction). As a result, in every case, the shape and in particular the maximum thickness of the free surface, located 
near the upper-left corner, are in much better agreement with the experiments.

The earlier instants (Figures 3(a), 3(b), 4(a), and 4(b)) are systematically the less accurate. A first reason is related to the free-slip boundary condition imposed on the uplifted gate in the model, whereas clear perturbations of the mass due to friction along the gate are observed in the first instants of the experimental collapse (see, e.g., Figures 14 and 15 in the work of Ionescu et al. ${ }^{24}$ ). The extents of the collapsing mass at intermediate times are pretty accurate and globally improved by including lateral friction. The shape of the mass is improved when including lateral friction even though at $\alpha=22^{\circ}$ some curvatures of the free surface are observed in the simulation and not in the experiments (Figures 4(c) and 4(d)). As detailed in Section VIII these oscillations do not appear when a constant viscosity is used in the model.

The shape of the mass near the front is not always sharply determined. This is mainly due to the size of the mesh cells compared to the aspect ratio of the domain (which goes from approximately 1 at the beginning to $1 / 50$ for the final deposit at $\left.\alpha=22^{\circ}\right)$. A higher refinement of the mesh would have produced a better description of the front zone. On the other hand, the experimental measurement of the front shape can be unreliable due to the saltation of the beads, leading to an error on the runout distances estimated to be $\pm 2 \mathrm{~cm}$ (see the work of Farin et $a l .{ }^{13}$ ).

\section{Dilatancy effects}

Another aspect of the granular collapse dynamics, generally omitted in the literature, is the strong dilatancy of the mass that occurs in the experiments. Figures 5 and 6 plot the volume variation as a function of time in the experiments and in the simulations for channel widths $w=10 \mathrm{~cm}$ and $w=20$ $\mathrm{cm}$, respectively. Note that volume variations in the simulations are numerical artefacts owing that incompressibility is assumed in the equations.

Despite the scattering of the measurements, we can see that the typical experimental behavior is an important volume increase at the beginning that decreases after some time to approximately retrieve the original compaction. In the case $w=10 \mathrm{~cm}$ (Figure 5), the experimental volume generally exhibits less than $3 \%$ of volume variation but locally reaches up to $5 \%$ of dilatancy (and compaction for $\alpha=22^{\circ}$ ). In every case, the final volume is almost equal to the initial one. These variations in volume partly explain the difficulty for the incompressible numerical simulation to reproduce the initial

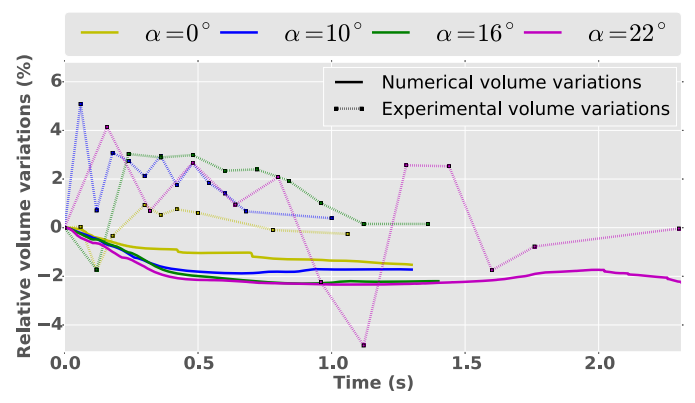

FIG. 5. Volume variations during time in the experiments and the numerical simulations for $w=10 \mathrm{~cm}$ for inclinations of the channel $\alpha=10^{\circ}, 16^{\circ}$, and $22^{\circ}$.

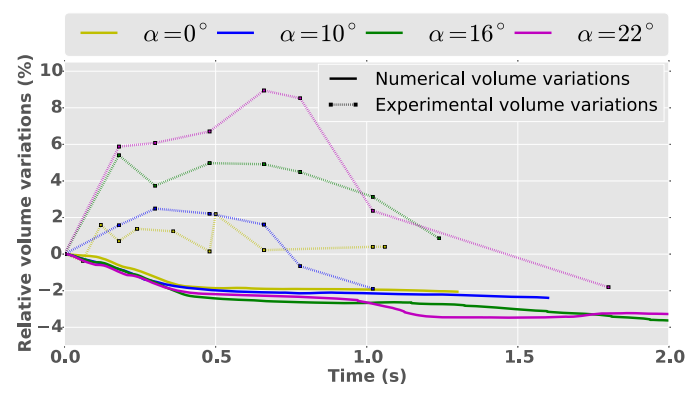

FIG. 6. Volume variations during time in the experiments and the numerical simulations for $w=20 \mathrm{~cm}$ for inclinations of the channel $\alpha=10^{\circ}, 16^{\circ}$, and $22^{\circ}$.

transient states with accuracy, especially since the numerical simulation tends to lose mass (around $2 \%$ at the end) when the domain quickly evolves (so basically during the acceleration phase). This numerical mass loss appears to be bigger on larger slopes. This observation suggests that an adaptive time scheme would be relevant to perform smaller time steps in the acceleration phase. We point out that another possibility for reducing mass loss is to consider a higher refinement of the mesh. We observed that the numerical mass loss linearly decreases with the mesh refinement (typically for a mesh size twice as small, the numerical mass loss is divided by 4 ).

In the case $w=20 \mathrm{~cm}$ (Figure 6), the variations of volume are stronger and strictly increase with the slope. For $\alpha=22^{\circ}$, the volume increase is close to $10 \%$. In addition, the volume of the final deposit is less close to the initial one than in the case $w=10 \mathrm{~cm}$. Note that in the case $\alpha=22^{\circ}$, the final mass seems more compact even though this might be due to experimental measurement errors. Conversely, the numerical mass behaves rather identically to the case $w=10 \mathrm{~cm}$. The velocities being slightly higher when $w=20 \mathrm{~cm}$, the mass loss seems to be slightly higher too but remains smaller than $3 \%$.

It is important to note that the use of a broader channel for the experiments, while providing reduced lateral wall effects, also allows for a stronger dilatancy to occur. In any case, a more accurate prediction of this type of experiment could only be achieved through the modeling of the dilatancy effects, for instance, through a dilatant Drucker-Prager model that takes into account the evolution of the volumic fraction with respect to the shearing of the material, e.g., the work of Andreotti et al. ${ }^{2}$

As a result, it appears insufficient to compare only the thickness profiles since their precision is limited by the sharpness of the mesh, the variations of volume, and the possibly variable precision of the experimental measurements. To take a closer look at the dynamical behavior, let us consider in the next part the evolution of the velocities of the front during the collapse.

\section{Front velocities}

Figures 7 and 8 represent the computed and experimental velocities of the front of the fluid domain during the collapse over slopes $\alpha=10^{\circ}, 16^{\circ}$, and $22^{\circ}$ with and without lateral friction for $w=10 \mathrm{~cm}$ and $w=20 \mathrm{~cm}$, respectively. The experimental front velocities have been simply calculated from the change in time of the measured positions of the front. The 


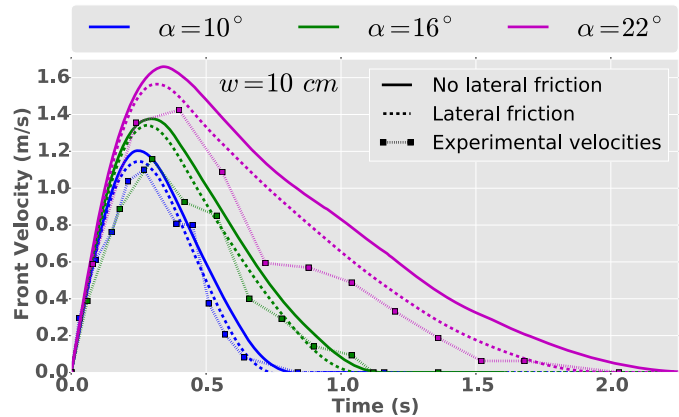

FIG. 7. Velocity of the front as a function of time computed with and without the lateral friction term and experimental velocities (obtained from the evolution of the position of the front with time) for $w=10 \mathrm{~cm}$ for inclinations of the channel $\alpha=10^{\circ}, 16^{\circ}$, and $22^{\circ}$.

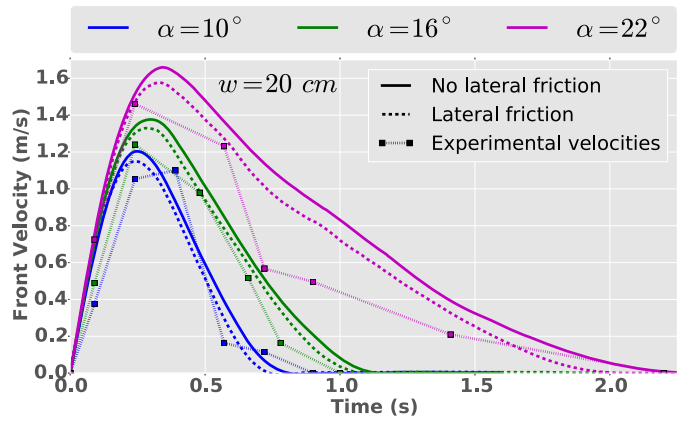

FIG. 8. Velocity of the front as a function of time computed with and without the lateral friction term and experimental velocities (obtained from the evolution of the position of the front with time) for $w=20 \mathrm{~cm}$ for inclinations of the channel $\alpha=10^{\circ}, 16^{\circ}$, and $22^{\circ}$.

curves consequently are made of few points with little precision on their position in time. It allows however us to observe the general behaviour.

In all cases, the velocities computed with lateral friction effects are clearly closer to the experimental front velocities. Typically, the maximum velocity is better reproduced when including lateral friction effect. At $22^{\circ}$, whatever the lateral friction and the channel width, simulations do not reproduce the two regimes observed in the experiments: a fast deceleration followed by the emergence of a slow propagation phase with a quite stable velocity for a given period of time (see the work of Mangeney et al. ${ }^{36}$ and Farin et al. ${ }^{13}$ ). In the simulations, the deceleration is on the contrary almost regular in time. As highlighted in Section VIII, the emergence of this slow propagation phase in the simulations depends on very fine changes of the rheological parameters.

\section{ROLE OF THE VALUE AND VARIABILITY OF THE VISCOSITY}

As explained in Section II, the $\mu(I)$ rheology only differs from a viscoplastic fluid with a Drucker-Prager plasticity criterion because of the spatio-temporally variable viscosity $\eta_{I}$ (see Equations (6) and (7)). Viscoplasticity has been shown to be of primary importance to achieve a good modelization of granular flows (e.g., the work of Lagrée et al. ${ }^{30}$ Andreotti et al. ${ }^{2}$ Silbert et al. ${ }^{48}$ and Jop et al. ${ }^{25}$ ). However, until now, little attention has been paid to the role of the variability of the viscosity $\eta_{I}$. Following the work of Ionescu et al. ${ }^{24}$ we will compare here the simulations obtained using $\eta_{I}$ and using a constant viscosity $\eta_{c}=0.4 \mathrm{~Pa}$ s. This value corresponds to a coarse spatio-temporal average of the range of values computed using $\eta_{I}$ for the collapses considered here (see Figure 11).

\section{A. Thickness profiles and front velocities}

Figure 9 plots the front velocities for various slopes computed with $\eta=\eta_{I}$ and $\eta=\eta_{c}$ (see Equations (6)-(8)) considering an infinitely wide channel $(w=\infty$ in (19)). Figure 10 plots the same results with $w=10 \mathrm{~cm}$.

In both cases $(w=\infty$ and $w=10 \mathrm{~cm})$, the differences between $\eta_{I}$ and $\eta_{c}$ are relatively small when looking at the acceleration and deceleration phases, the maximum velocity, and the stopping time. The simulations with the viscosity $\eta_{I}$ do not predict any peculiar behavior that the constant viscosity model fails to predict. As a matter of fact, not only the front velocities but also the dynamical shape of the domain is hardly affected by the variable viscosity. Figure 11 shows that the contour of the domains computed with and without a variable viscosity is very similar for different slopes even if the viscosity field varies significantly within the domain. These contours are represented at the time corresponding to the maximum velocity where the inertial number strongly varies within the fluid domain (typically $I$ varies between $10^{-3}$ close to the static-flowing transition and 1 at the surface and the front, see the work of Ionescu $e t a l .{ }^{24}$ ). Note that the constant viscosity $\eta_{c}$ does not produce the oscillations of the free surface that

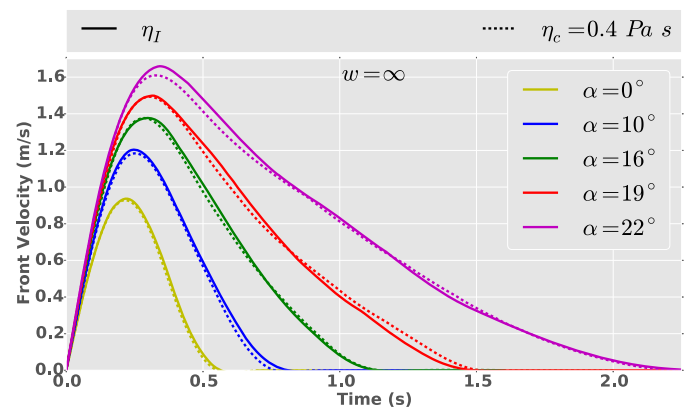

FIG. 9. Velocity of the front as a function of time for $\alpha=0^{\circ}, 10^{\circ}, 16^{\circ}, 19^{\circ}$, and $22^{\circ}$ computed with $\eta_{I}$ viscosity and constant viscosity $\eta_{c}=0.4 \mathrm{~Pa}$. The value for the constant viscosity $\eta=0.4 \mathrm{~Pa}$ s is chosen as an average value of the viscosity obtained from Equation (6) in the flowing part of the fluid domain. The velocities have been computed without lateral friction effects $(w=\infty)$.

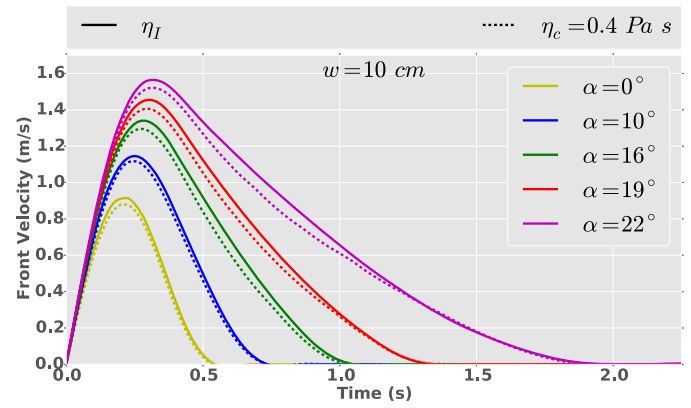

FIG. 10. Velocity of the front as a function of time for $\alpha=0^{\circ}, 10^{\circ}, 16^{\circ}, 19^{\circ}$, and $22^{\circ}$ computed with $\eta_{I}$ viscosity and constant viscosity $\eta_{c}$. The velocities have been computed with $w=10 \mathrm{~cm}$. 


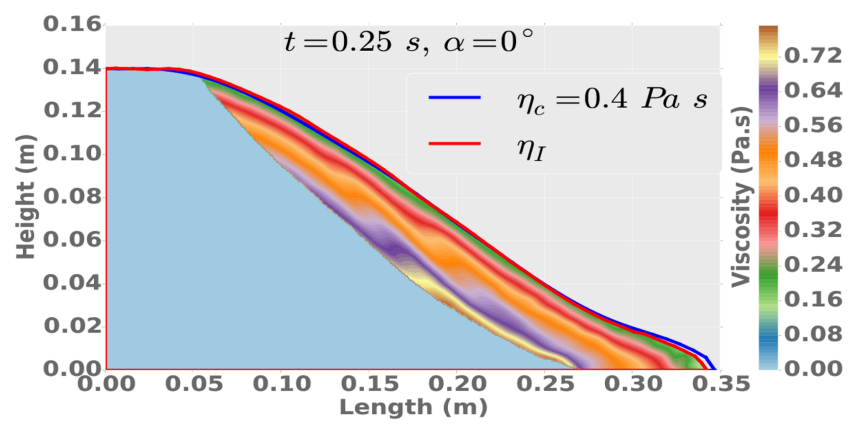

(a) $\alpha=0^{\circ}$

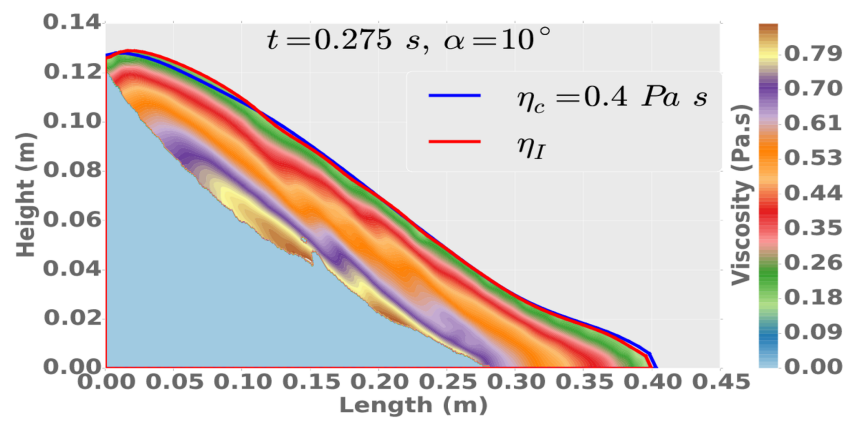

(b) $\alpha=10^{\circ}$

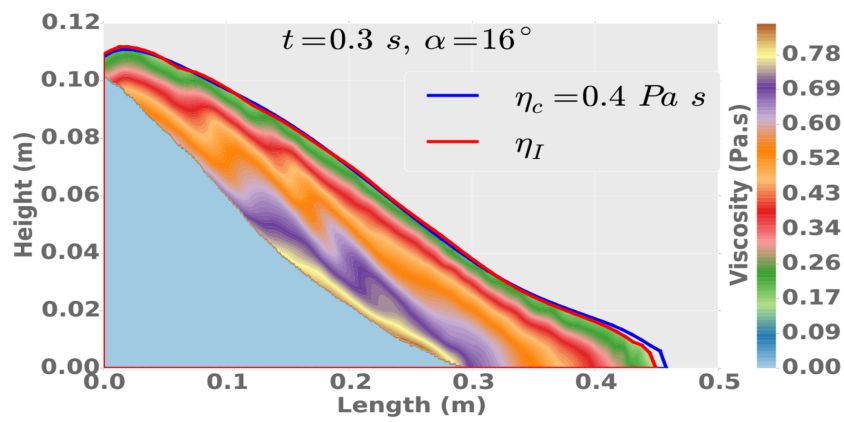

(c) $\alpha=16^{\circ}$

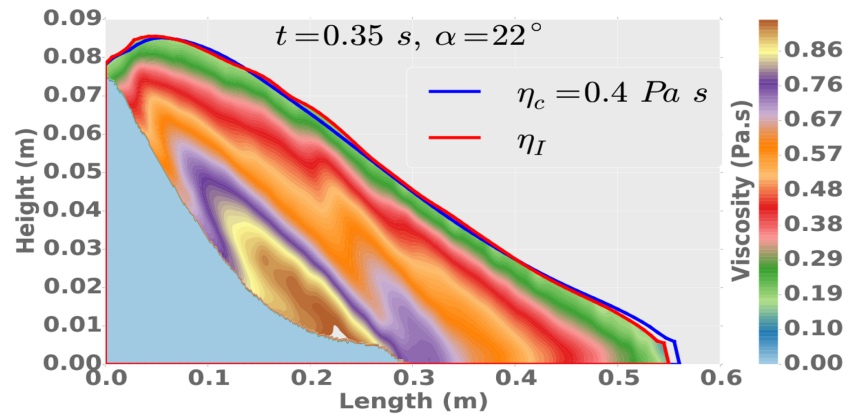

(d) $\alpha=22^{\circ}$

FIG. 11. Computed thickness profiles for inclinations of the channel $\alpha=0^{\circ}$, $10^{\circ}, 16^{\circ}$, and $22^{\circ}$ computed with $\eta_{I}$ and $\eta_{c}$. The color field displays the value of the viscosity $\eta_{I}$ in the flowing part (see Equation (6)). The chosen times correspond to the time of maximum front velocity for each slope. The velocities have been computed with $w=10 \mathrm{~cm}$. The viscosity field in the static part has been set to 0 for clarity.

appear with $\eta_{I}$ at intermediate times for slope angle $\alpha=22^{\circ}$ (see Figure 17). As a matter of fact, perturbation of the free surface with $\eta_{I}$ at large slopes is systematic and is not observed with a constant viscosity $\eta_{c}$. Note that the low sensitivity of the model to the spatial variability of the viscosity has been highlighted for other non-Newtonian fluids (see, e.g., the work of Martin and Monnier ${ }^{38}$ ).

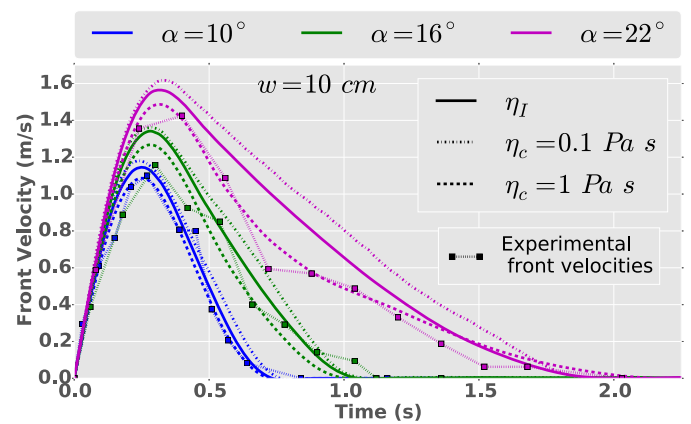

FIG. 12. Computed and experimental velocities of the front as a function of time for $w=10 \mathrm{~cm}$ and for inclinations of the channel $\alpha=10^{\circ}, 16^{\circ}$, and $22^{\circ}$ computed with $\eta_{I}$ and $\eta_{c}$. The values $\eta_{c}=0.1 \mathrm{~Pa} \mathrm{~s}$ and $\eta_{c}=1 \mathrm{~Pa}$ s have been chosen as the bounds of the viscosity range provided by $\eta_{I}$ (see Figure 11).

The observable differences between a constant and variable viscosity mainly come from the fact that the value of $\eta=0.4 \mathrm{~Pa} \mathrm{~s}$ is slightly higher in general than the arithmetic average of the viscosity computed by Equation (6) leading to a slightly slower flow. The differences are however stronger when lateral friction is taken into account, possibly due to the non-linear coupling between this friction and the velocity field.

While the spatio-temporal variability of the viscosity does not have a significant influence on the flow, the absolute value of the viscosity has. This is illustrated in Figures 12 and 13 representing the results obtained with $\eta_{I}$ and different values of $\eta_{c}$ for different slopes and for $w=10 \mathrm{~cm}$ and $w=20 \mathrm{~cm}$, respectively. A higher viscosity leads to a smaller maximum velocity but the slope of the velocity curves in the first part of the acceleration phase is almost identical. They start to differ from one another before the velocity peak. The value $\eta_{c}=1$ Pa s provides the best fit to the experiments.

During the deceleration phase, the behavior is significantly modified by changing the value of $\eta_{c}$. It appears that the convexity of the curve is strongly related to the value of $\eta_{c}$. A smaller value leads to an almost constant deceleration whereas a higher value induces a change of slope in the velocity curve. The differences remain small at small slopes. For $\alpha=22^{\circ}$, the value $\eta_{c}=1 \mathrm{~Pa}$ s predicts two different regimes in the deceleration phase: first, a quick deceleration followed by what can be seen as a slow propagation phase. To assess the sensitivity of the slow propagation phenomenon to the value of the viscosity, we plot the results obtained with $\eta_{I}, \eta_{c}=1$

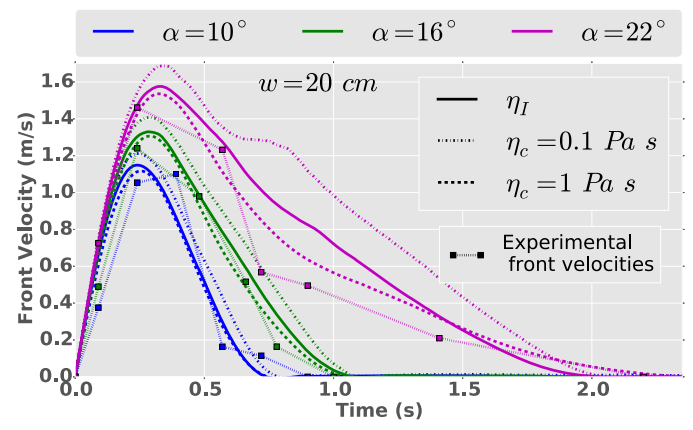

FIG. 13. Computed and experimental velocities of the front as a function of time for $w=20 \mathrm{~cm}$ and for inclinations of the channel $\alpha=10^{\circ}, 16^{\circ}$, and $22^{\circ}$ computed with $\eta_{I}$ and $\eta_{c}$. The values $\eta_{c}=0.1 \mathrm{~Pa} \mathrm{~s}$ and $\eta_{c}=1 \mathrm{~Pa}$ s have been chosen as the bounds of the viscosity range provided by $\eta_{I}$ (see Figure 11). 


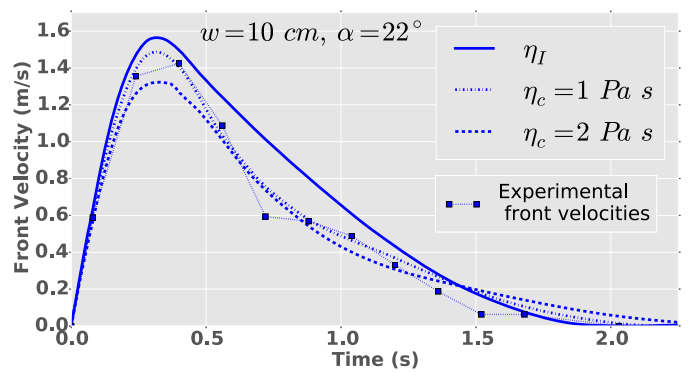

FIG. 14. Computed and experimental velocities of the front as a function of time for $w=10 \mathrm{~cm}$ and for $\alpha=22^{\circ}$ computed with $\eta_{I}, \eta_{c}=1 \mathrm{~Pa} \mathrm{~s}$ and $\eta_{c}=2 \mathrm{Pas}$.

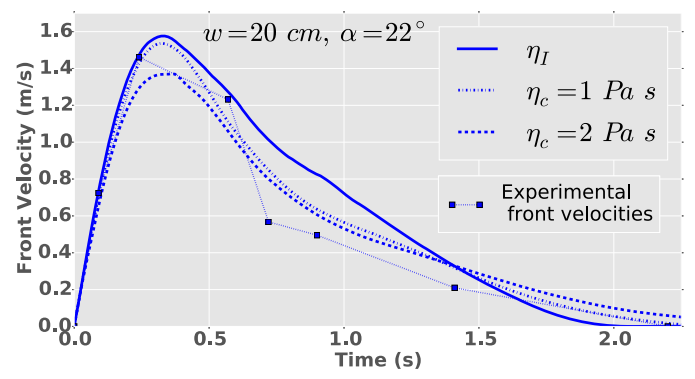

FIG. 15. Computed and experimental velocities of the front as a function of time for $w=20 \mathrm{~cm}$ and for $\alpha=22^{\circ}$ computed with $\eta_{I}, \eta_{c}=1 \mathrm{~Pa} \mathrm{~s}$ and $\eta_{c}=2 \mathrm{Pas}$

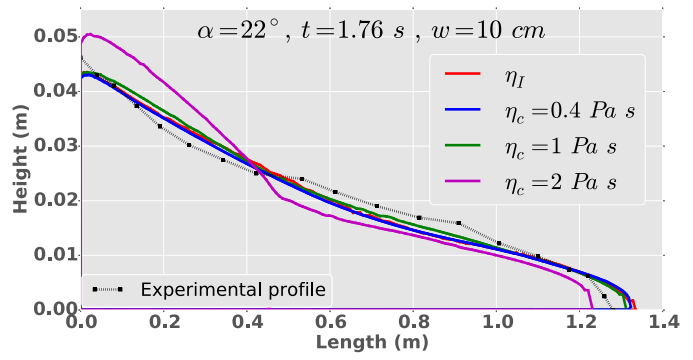

FIG. 16. Computed and experimental thickness profiles for an inclination of the channel for $w=10 \mathrm{~cm}$ and for $\alpha=22^{\circ}$ computed with $\eta_{I}, \eta_{c}=0.4 \mathrm{~Pa} \mathrm{~s}$ $\eta_{c}=1 \mathrm{~Pa} \mathrm{~s}$ and $\eta_{c}=2 \mathrm{~Pa} \mathrm{~s}$.

Pa s and $\eta_{c}=2 \mathrm{~Pa} \mathrm{~s}$ in Figures 14 and 15 for $\alpha=22^{\circ}$, for $w$ $=10 \mathrm{~cm}$ and $w=20 \mathrm{~cm}$, respectively.

The slow propagation phase lasts longer when $\eta_{c}=2 \mathrm{~Pa}$ $\mathrm{s}$. The duration and shape of this slow phase is better reproduced with $\eta_{c}=1 \mathrm{~Pa}$ s. We plot in Figure 16 the final deposits computed with $\eta_{I}, \eta_{c}=0.4 \mathrm{~Pa} \mathrm{~s}, \eta_{c}=1 \mathrm{~Pa} \mathrm{~s}$, and $\eta_{c}=2 \mathrm{~Pa} \mathrm{~s}$. As we can see, the differences between the deposits are small (except for $\eta_{c}=2 \mathrm{~Pa} \mathrm{~s}$ ) and one cannot tell if the deposit obtained with $\eta_{c}=1 \mathrm{~Pa}$ s is closer to the experimental profile than the others. It results that, for $\eta_{c} \in[0.4,1] \mathrm{Pa}$ s, while the final deposit is barely affected by the value of the viscosity, the dynamics experiences significant changes.

Our simulations suggest that the slow propagation phase is related to the subtle balance between the mean viscosity of the flow and the other adjustable physical quantities of the model, namely, the internal and boundary friction coefficients. Calibration of the viscosity on the velocity front variations seems thus to be very precise. Indeed, for every angle, the front velocity curves obtained with $\eta_{c}=1 \mathrm{~Pa}$ s much better agrees with the experimental front velocities in all the phases of the flow (acceleration, deceleration, slow propagation). Note that the very good agreement between the simulation with $\eta_{c}=1 \mathrm{~Pa}$ s and the experimental front velocities suggests that the maximum velocity has been missed in the experimental measurements (Figures 12 and 13).

\section{B. Pressure fields}

The pressure is a crucial quantity here since it defines the position of the static-flowing interface through the DruckerPrager plasticity criterion. A classical simplification of these granular flows, typically for large scale realistic simulations, is to consider the pressure to be hydrostatic. However, the pressure within a Drucker-Prager flow has a priori non-hydrostatic components. In the recent work, Bouchut et al. ${ }^{6}$ derived nonhydrostatic correction terms from the Drucker-Prager model with constant viscosity $\eta_{c}$ based on the shallow flow approximation developed up to second order. More precisely, the shallow approximation assumes that the thickness, stresses, and pressure are of order $\varepsilon$ and the flow is slow, i.e., the streamwise velocity is of order $\varepsilon$. The resulting analytical pressure reads in the topography related frame $(X, Z)$,

$$
\begin{aligned}
p= & \rho g\left(\cos \alpha-\sin \alpha \partial_{X} h\right. \\
& \left.-2|\sin \alpha| \frac{\partial_{X} U}{\left|\partial_{Z} U\right|}\right)(h-Z)+\mathcal{O}\left(\varepsilon^{3}\right),
\end{aligned}
$$

where $(U, W)$ is the velocity field and $h(X)$ is the height of the free-surface (in the topography related frame $(X, Z)$ ). The two terms describing the deviation from hydrostatic pressure are related to the surface slope effect and to streamwise velocity gradients, respectively. Note that the last term in Equation (22) is only defined in the flowing phase. The expression of (22) in the gravity related frame $(x, z)$ considered hereafter is given in Appendix B.

In order to further investigate the effect of the variable viscosity $\eta_{I}$, we compare hereafter the computed pressure profiles on a slope $\alpha=22^{\circ}$ obtained with $\eta_{I}$ and $\eta_{c}$ to the analytical pressure given in (22). In order to have a rather shallow problem with a relatively small average velocity consistent with the hypothesis of the analytical pressure, we consider the results at time $t=1.5 \mathrm{~s}$ where the flow is fully developed. The domain is plotted in Figure 17. The resulting pressure profiles are plotted in Figure 18.

A first observation is that the deviation from the hydrostatic pressure is relatively small. However, accounting for the non-hydrostatic terms significantly improves the precision of the calculated pressure and allows us to capture quite well the pressure variation with depth. The analytical pressure (22), derived from the constant viscosity Drucker-Prager model, allows, with an identical precision, us to describe the pressure field computed with both the constant and variable viscosity.

Since the fluid domains computed with $\eta_{c}$ and $\eta_{I}$ are slightly different, the pressure profiles at a given abscissa $x$ are obviously not the same. However, overall, it does not seem that the variable viscosity predicts a pressure field radically different from that obtained with the constant viscosity. 


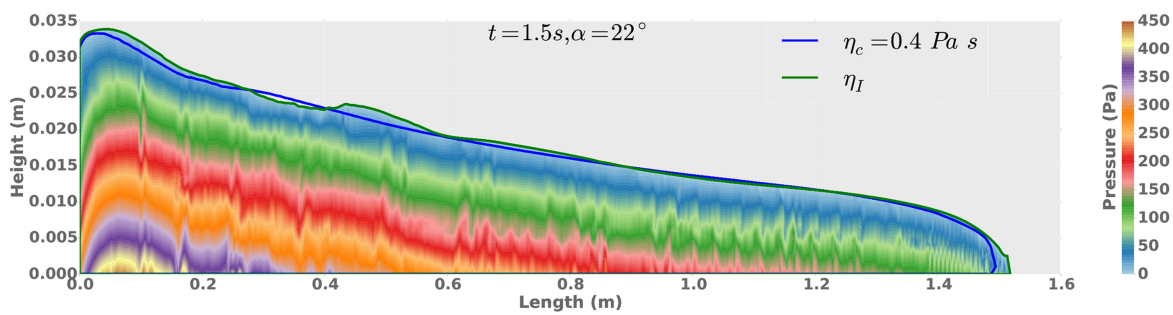

FIG. 17. Thickness profiles of the granular collapse computed for $\alpha=22^{\circ}$ with variable $\eta_{I}$ and constant $\eta_{c}$ viscosities at $t=1.5 \mathrm{~s}$. The colored surface represents the pressure field computed with $\eta_{I}$. It is worth noting that the real aspect ratio of the plot is the tenth one as small as the plotted one (hence the perturbed aspect of the pressure field).

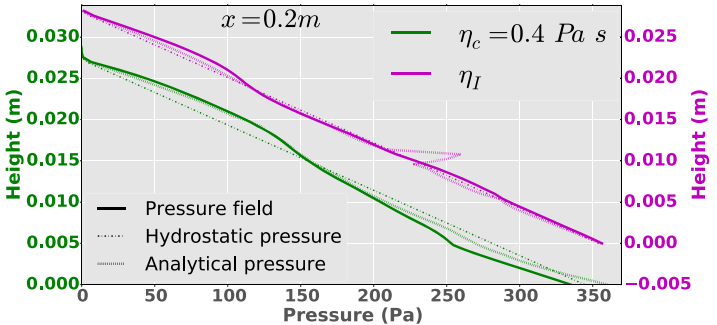

(a) $x=0.2 \mathrm{~m}$

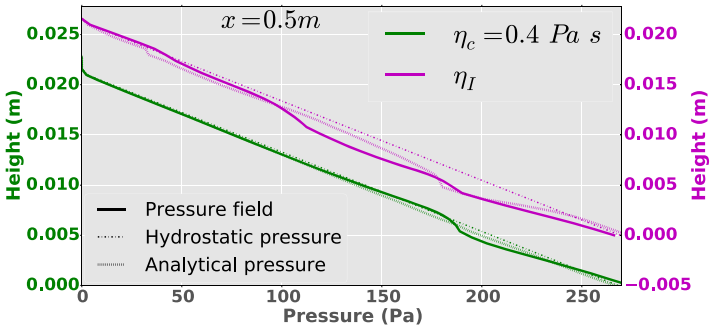

(c) $x=0.5 \mathrm{~m}$

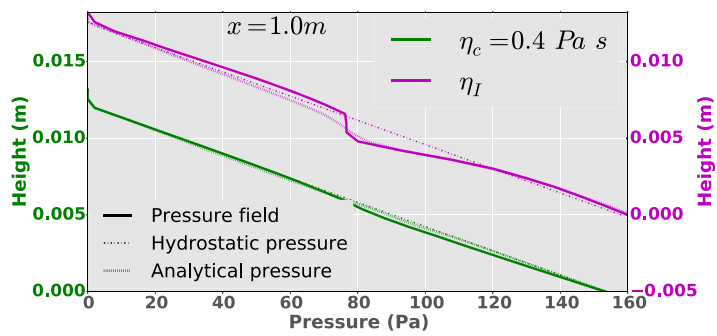

(e) $x=1 \mathrm{~m}$

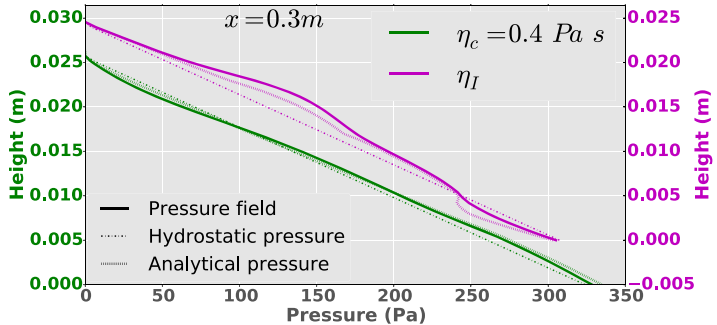

(b) $x=0.3 \mathrm{~m}$

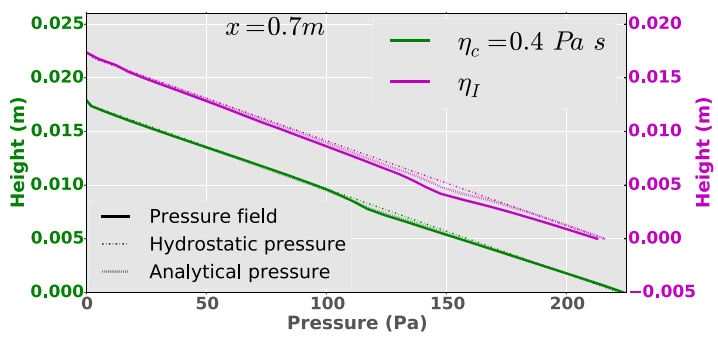

(d) $x=0.7 \mathrm{~m}$

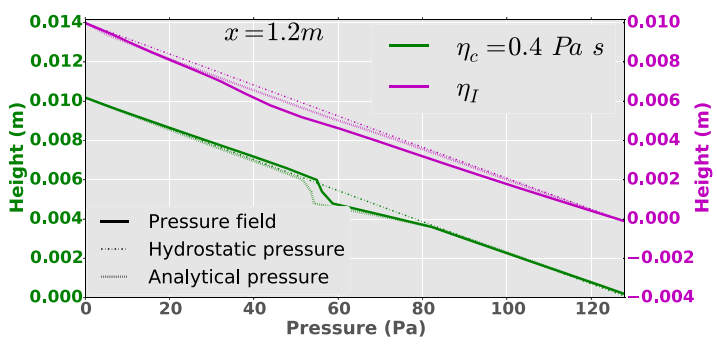

(f) $x=1.2 \mathrm{~m}$

FIG. 18. Pressure profiles at different abscissae extracted from the pressure field computed with $\eta_{I}$ and plotted in Figure 17 and its counterpart computed with $\eta_{c}$, analytical pressure evaluated from (22), and corresponding hydrostatic pressure. The profiles in magenta are plotted using the right y-axis. The plotted profiles are vertical cuts of the domain plotted in Figure 17.

\section{Summary}

In the present case, we have seen that the variability of the viscosity $\eta_{I}$ derived from the $\mu(I)$ rheology is of very little effect when compared to the results obtained with a constant viscosity model with the same value of viscosity in average. On the other hand, we have demonstrated that the flow dynamics is very sensitive to the value of the viscosity (and not to its spatio-temporal variation). In particular, simulating the front velocity makes it possible to precisely calibrate the value of the viscosity. The average viscosity obtained from the $\mu(I)$ rheology provides a very good order of magnitude of the best fitted viscosity. However, with the physical parameters considered here, this value is too small. Here the viscosity $\eta_{c}=1$ $\mathrm{Pa}$ s, which is within the upper values of the $\eta_{I}$ viscosity, gives better agreement with the experiments. It allows, in particular, us to capture the slow propagation phase which was not possible with the variable viscosity considered here.

\section{ANALYSIS AND IMPACT OF SMALL SCALE MODEL-INSTABILITIES}

This section focuses on the presence of small scale instabilities (also called ill-posed behavior) associated with the mechanical model. The main goal is to analyse and assess how these instabilities affect the numerical simulation and more generally their impact on the modeling of granular flows.

Very recently Barker et $\mathrm{al}^{3}$. have studied the "wellposedness" of the $\mu(I)$ rheological model through a linear 
stability analysis. The terminology "well-posed" or "ill-posed" problem, used by them to distinguish between stable or unstable processes, respectively, is related to the old definition of Hadamard ${ }^{18}$ who considered a problem to be well-posed if it has a unique and stable solution. In what follows, we use both terminologies to discriminate between linearly stable and unstable behaviors.

Starting from a reference flow, Barker et al. ${ }^{3}$ establish a (linear) stability criterion: if in some region of the reference flow, the inequalities (2.40)-(2.41) of their paper (or equivalently (2.43)) are satisfied, then one can expect an unbounded growth of short-wavelength perturbations, i.e., the problem is ill-posed. Note that the stability condition is computed locally in time and space from the reference flow. Only small-scale perturbations are affected, hence the model-instability could be detected numerically only for very fine meshes. This is why we would like to analyse here the mesh dependency of the flow, starting from a "normal" mesh computation as reference flow.

Since we would like to evaluate the influence of the $\mu(I)$ viscosity on small-scale instabilities, we consider in addition the case of constant viscosity (Drucker-Prager fluid). A linear stability analysis of the Drucker-Parger flow, similar to the one done by Barker et al. ${ }^{3}$ (see also the work of Schaeffer ${ }^{49}$ and Schaeffer and Pitman ${ }^{50}$ ) can be found in Appendix C. We get the ill-posedness (instability) criterion (C12) with (C11) to be satisfied for getting an unbounded growth of short-wavelength perturbations.

We would like to prove here that the presence of smallscale instabilities is inherent to the choice of the incompressible Drucker-Prager plasticity model (non-associated plasticity) and not to secondary effects as variable viscosity $(\mu(I)$-rheology), lateral wall friction, or barrier modeling. This is the reason why, in what follows, we choose to present two cases. The first one has been studied before: $\mu(I)$-rheology with lateral wall effect and gate modeling. For the second one, we consider a Drucker-Prager fluid with constant viscosity $\eta_{c}=0.4 \mathrm{~Pa}$ s without any lateral-wall or gate effects. The reference flow for both cases will be the numerical computation with a normal mesh (called coarse mesh in this section) with a mesh size $h=0.008 \mathrm{~m}$ (corresponding to approximatively 1000 triangle cells). In Figure 19 we have plotted in orange the stable (well-posed) regions at time $t=0.1 \mathrm{~s}$. They have been computed from the criteria (2.40)-(2.41) of Barker et al. ${ }^{3}$ for the $\mu(I)$ - rheology (left side) and from (C12) for the DruckerPrager fluid (right side). We remark that in both cases almost all the flow region is unstable (ill-posed). Between these two ill-posed (unstable) models, the $\mu(I)$-rheology presents a larger stability region.

We first analyze the case of the $\mu(I)$-rheology. We plot in Figure 20 the norm and iso-contours of the computed velocity field and of the corresponding strain-rate field at time $t=0.1 \mathrm{~s}$ on a $0^{\circ}$ slope for the reference flow (mesh sizes $h=0.008 \mathrm{~m}$ ), and for $h=0.004 \mathrm{~m}$ and $h=0.002 \mathrm{~m}$ (corresponding to approximatively 5000 and 20000 triangular cells, respectively). As we can see on the coarser mesh (Figure 20(a)), the isolines are basically evenly spaced according to the value of the velocity field. As we refine the mesh, the velocity field becomes stratified and the bands of high velocity gradient appear. On the finer mesh, the short-wave model-instability is very clear and the velocity field almost appears as a sequence of plug flows separated by thin zones of high gradient. This behavior is clearly retrieved on the strain rate field plotted in Figures 20(d)-20(f), where the color scale related to strain rate has been saturated for the sake of readability to a maximum of $40 \mathrm{~s}^{-1}$. Some "shear bands" appear at the interfaces between the plug zones. This behavior is not related to a physical instability since there is no convergence to a given number of shear bands when the mesh size decreases (up to the smallest tested mesh size, i.e., $\mathrm{h}=0.001 \mathrm{~m}$ for shear bands width of around 3-4 grain diameters). These fictitious "shear bands" always cover a thickness of two to three elements, which is the smallest size for a short-wave instability which could be captured by a finite element computation.

We can perform the same analysis for the second case, the Drucker-Prager fluid with a constant viscosity $\eta_{c}=0.4 \mathrm{~Pa} \mathrm{~s}$ and without any lateral-wall or gate effects. In Figure 21 the norm and iso-contours of the computed velocity and strain rate fields are plotted at time $t=0.1 \mathrm{~s}$ on a $0^{\circ}$ slope for the reference flow (mesh sizes $h=0.008 \mathrm{~m}$ ) and for $h=0.004 \mathrm{~m}$ and $h=0.002 \mathrm{~m}$. As for the $\mu(I)$-rheology model, when we refine the mesh the velocity field becomes stratified and the bands of high gradient occur for the finer mesh, the shortwave model-instability appears through a sequence of plug flows separated by thin zone of high gradient. As before the "shear bands" always cover a thickness of two to three elements which is consistent with the stability analysis given in Appendix C.

These results highlight the fact that the model-instabilities (model ill-posedness) are present even in modeling usual and simple granular flows, as the column collapse studied here. The model instabilities are not avoided by considering

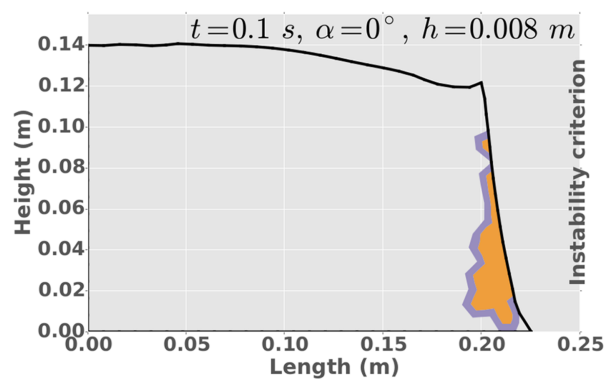

(a)Stable (well-posed) regions for the $\mu(I)$-rheology

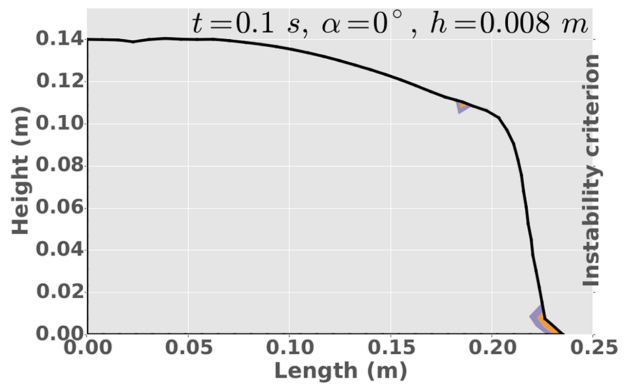

(b)Stable (well-posed) regions for the Drucker-Prager fluid
FIG. 19. The stable regions (in orange) at time $t=0.1 \mathrm{~s}$ computed from the criteria (2.40)-(2.41) of Barker et al. ${ }^{3}$ for the $\mu(I)$ - rheology (left side) and from (C12) for the Drucker-Prager fluid (right side). 


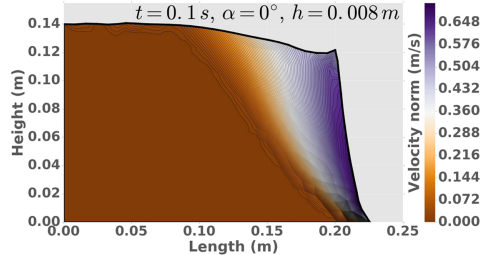

(a)Velocity norm, $h=0.008 \mathrm{~m}$

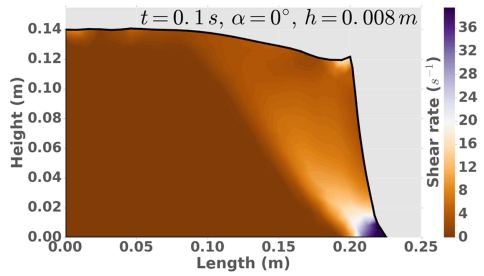

(d)Strain rate, $h=0.008 \mathrm{~m}$

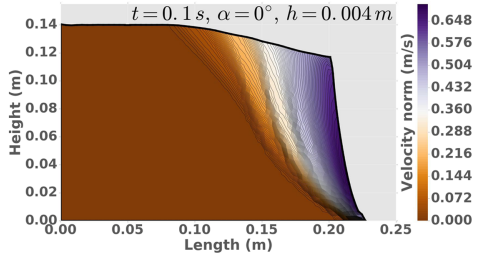

(b) Velocity norm, $h=0.004 \mathrm{~m}$

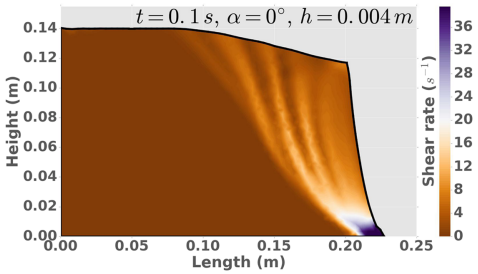

(e)Strain rate, $h=0.004 \mathrm{~m}$

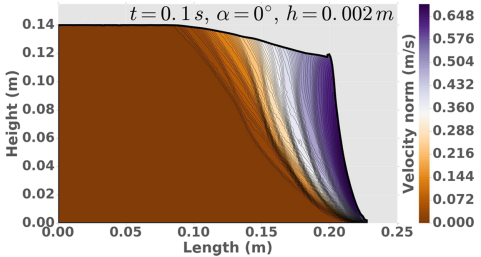

(c) Velocity norm, $h=0.002 \mathrm{~m}$

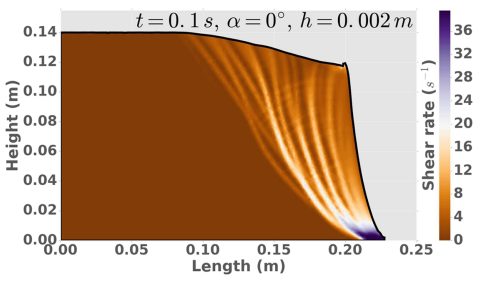

(f)Strain rate, $h=0.002 \mathrm{~m}$

FIG. 20. Short-wave model-instability of the $\mu(I)$-rheology. Velocity norm (up) and strain rate (down) computed at time $t=0.1 \mathrm{~s}$ on a slope $\alpha=0{ }^{\circ}$ on meshes with cells of size $h=0.008$ (left), $h=0.004$ (middle), and $h=0.002$ (right).

variable viscosity models, as the $\mu(I)$-rheology. It seems that they are related to the use of the Drucker-Prager plasticity criterion (1) in the context of an incompressible flow. Indeed, for the von Mises plasticity (i.e., the Bingham model), the incompressibility condition is a consequence of the maximum plastic power dissipation principle, valid for the so called "associated plasticity models." For these models we can associate a plastic potential and give an energetic variational principle. If we want to use the Drucker-Prager criterion (1) in the context of associated plasticity, then we obtain a rather complicated compressible model (see the work of Cazacu and Ionescu, ${ }^{7}$ section "It does not always work"). The model including the incompressibility assumption and the Drucker-Prager plasticity criterion (1) does not belong to the family of associated plasticity models and there is no associated plastic potential. This could be an explanation for the presence of model instabilities in describing granular flows.

However, the numerical simulations show that the instability does not affect the overall results in terms of dynamical shape or static-flowing transition. As an example, Figure 22 plots the thickness profiles of the second case (Drucker-Prager fluid with a constant viscosity $\eta_{c}=0.4 \mathrm{~Pa}$ $\mathrm{s}$ and without any lateral-wall or gate effects) at time $t=0.5 \mathrm{~s}$ for the three mesh sizes. From this figure, we clearly observe that the thickness profiles are not modified when refining the mesh, and the differences that can be observed between the three profiles are completely expected, regarding the higher precision one gets from a finer mesh. The velocity range is not affected by the fictitious shear bands and only its spatial structure is. We conclude that the model and the associated computations are robust for "normal" mesh size, where no short-wave instabilities are present. The mesh refining technique is essential for "continuous-type" models but it has some limits in modeling granular materials which have a small scale characteristic length. It suggests that the model could eventually be "regularized" by the introduction of a small parameter, the grain diameter. Indeed for our continuous visco-plastic model, the short wave instabilities are

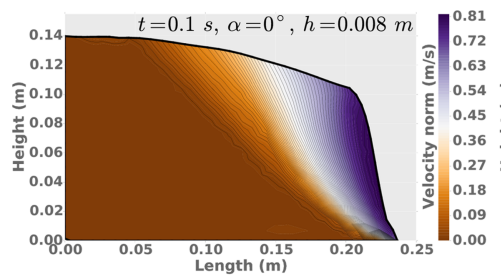

(a) Velocity norm, $h=0.008 \mathrm{~m}$

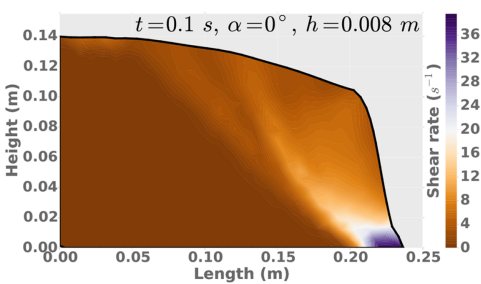

(d)Strain rate, $h=0.008 \mathrm{~m}$

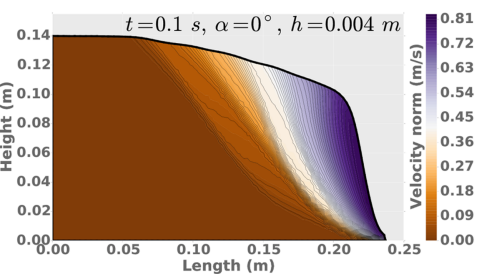

(b) Velocity norm, $h=0.004 \mathrm{~m}$

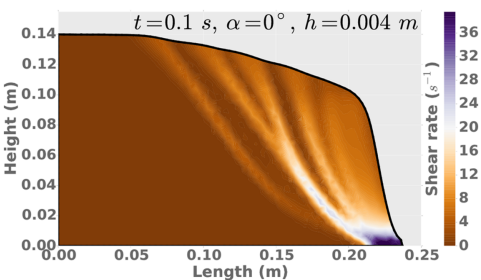

(e)Strain rate, $h=0.004 \mathrm{~m}$

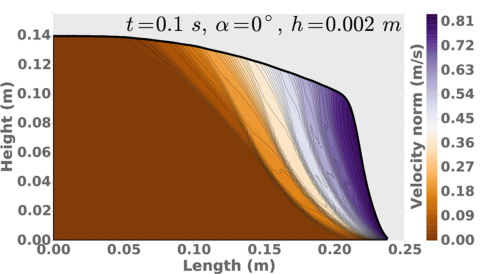

(c) Velocity norm, $h=0.002 \mathrm{~m}$

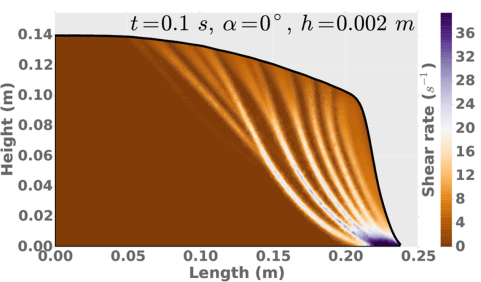

(f)Strain rate, $h=0.002 \mathrm{~m}$

FIG. 21. Short-wave model-instability of the Drucker-Prager fluid (constant viscosity). Velocity norm (up) and strain rate (down) computed at time $t=0.1 \mathrm{~s}$ on a slope $\alpha=0^{\circ}$ on meshes with cells of size $h=0.008$ (left), $h=0.004$ (middle), and $h=0.002$ (right). 


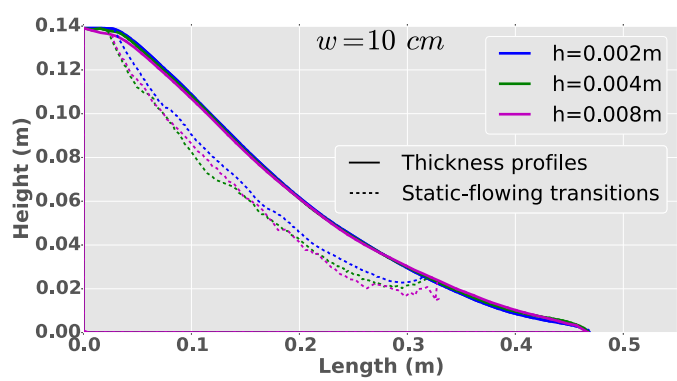

FIG. 22. Thickness profiles computed with increasingly fine meshes at time $t=0.5 \mathrm{~s}$ on a $0^{\circ}$ channel and the corresponding static-flowing transitions.

present only when the mesh size is of order of a few grain diameter. At this level of mesh refinement, the continuous (finite element) model might be no longer computationally attractive and replaced by a discrete-element numerical approach.

\section{DISCUSSION AND CONCLUSION}

Following the work of Ionescu et al. ${ }^{24}$ we have compared quantitatively the simulation of a granular column collapse using a continuum viscoplastic model with laboratory experiments. One of the main focuses of this paper is to introduce lateral wall friction in a 2D model and quantify its effect. Confinement and subsequent lateral friction may occur in natural landslides or debris avalanches when the flow is channelized into valley walls. However as these natural flows are generally three dimensional, they can barely be simulated by 2D flows. The lateral wall issue is mainly addressed here to be able to compare quantitatively simulation and laboratory experiments of granular flows in a channel.

Our results show that precise quantitative agreement with the dynamics and deposit of granular column collapse over inclined planes requires us to take into account the lateral wall effects that were poorly handled in Ref. 24. By assuming a constant flow in the channel width direction, a Coulomb friction on the lateral walls is rigorously introduced in the variational formulation and, following the augmented Lagrangian approach and the regularization used for the Coulomb friction on the boundaries, solved likewise (see Appendix A). The resulting solutions significantly improve the former ones without any adjustments on the parameters and allow us to achieve accurate simulations of granular column collapse on a wide range of slopes (from $0^{\circ}$ to $22^{\circ}$ ). The transient thickness profiles are significantly closer to the observed ones when simulated with lateral friction and provide a quite faithful tracking of the spreading of the granular mass. In particular, lateral friction prevents the mass from the upper-left corner to flow downstream too much. The simulated runout distances lie within a $5 \%$ accuracy compared to the experimental ones which are estimated to be precise at $\pm 2 \mathrm{~cm}$. It follows that the simulated runout distances for $\alpha=10^{\circ}$ and $\alpha=16^{\circ}$ are closer to the observation than the measurement precision. For $\alpha=22^{\circ}$, the difference between the simulated and the observed runout distance is around twice the measurement precision. The improvement is particularly significant at large slopes. This result is all the more significant than realistic geophysical granular flows which mostly occur on slopes higher than $16^{\circ}$.
Higher slopes could likely be simulated with the present model and the main limitation is that, the fluid domain becoming very shallow for slopes larger than $22^{\circ}$, a highly refined mesh is necessary to achieve such simulations without losing precision.

The ability of the model to capture the slow propagation phase suggests that its ingredients represent quite well the physical processes involved. In the present case, we observe that, when calibrating the viscosity, a constant viscosity Drucker-Prager model is able to reproduce this slow velocity phase. In our case, we show that the spatio-temporal variability of the viscosity $\eta_{I}$ does not induce remarkable differences of behavior (thickness, velocity, pressure) compared to a constant viscosity model. The observed front velocity during the collapse proves to be a reliable tool to adjust the value of the constant viscosity in the model. The question is that what is the physical meaning of this viscosity and as to whether it can be measured directly in the experiments.

The present study and that of Ionescu et al. ${ }^{24}$ show that quantitative simulation of granular collapses over inclined planes could be only achieved by accounting for the effects of (1) lateral wall friction and (2) the gate. As a free-slip boundary condition on the uplifting gate was considered here and in Ref. 24, a more accurate simulation would require to model the friction occurring between the granular mass and the gate. Furthermore, the flow is not constant in the $Y$-direction as assumed here. Taking into account the $3 \mathrm{D}$ effects leading to smaller velocities near the walls than within the center of the channel may also improve the quantitative agreement with the experimental data.

From a numerical point of view, simulation of the front, of the collapse near the upper-left corner, and of mass conservation would be improved by higher mesh refinement and adaptative time step.

However, going further in reducing the error between simulation and experiments requires us to develop models that take into account dilatancy effects. Indeed, the present work demonstrates that dilatancy is observed in the experiments, in particular, a volume increase at the beginning of the collapse. This dilatancy can reach up to $10 \%$ on slopes $\alpha=22^{\circ}$. Note that dilatancy effects are stronger for collapses in a broader channel.

We have analyzed how the small scale instabilities (also called ill-posed behavior) associated with the mechanical model affect the numerical simulation. These model instabilities, characterized by Barker et al. $^{3}$ (see also the work of Schaeffer, ${ }^{49}$ Schaeffer and Pitman, ${ }^{50}$ and Appendix C), are present even in modeling usual and simple granular flows, as the column collapse studied here.

When we refine the mesh, the velocity field becomes stratified and the bands of high gradient in the velocity field appear. These fictitious "shear bands" are not related to a physical instability and cover a thickness of two to three elements (the smallest size of a short-wave instability captured by a finite element technique). The model instabilities are not avoided by considering variable viscosity models, as the $\mu(I)$ - rheology. The existence of the small-scale instabilities seems to be inherent to the choice of incompressible Drucker-Prager plasticity model (non-associated plasticity). 
The short-wave instability does not affect the overall results as the fluid shape, the static-flowing transition, or the velocity range. The triggering of these short-wave instabilities occurs only for a mesh size of order of a few grain diameters.

\section{ACKNOWLEDGMENTS}

This work has been partially funded by the USPC project PAGES, CNCS-UEFISCDI Project No. PN-II-ID-PCE-20113-0045, and by the ERC Contract No. ERC-CG-2013-PE10617472 SLIDEQUAKES.

\section{APPENDIX A: NUMERICAL METHOD}

We describe here the numerical algorithm used to solve the dynamic flow problem described above. This numerical method is an extension of the one presented in the work of Ionescu et al. ${ }^{24}$ to include the lateral friction effects. Appendix A 1 briefly presents the method and its modification and we refer to the work of Ionescu et al. ${ }^{24}$ for a more comprehensive description.

The time discretization is achieved using an implicit Euler scheme, and a set of nonlinear equations on the velocity $\boldsymbol{u}$, the deviatoric stress tensor $\boldsymbol{S}$, and the pressure $p$ is to be solved at each time step.

To overcome the difficulties related to the nondifferentiability of the viscoplastic and friction terms, an iterative decomposition-coordination formulation coupled with the augmented Lagrangian method of Glowinski and Le Tallec ${ }^{17}$ and Fortin and Glowinski ${ }^{14}$ is adapted here. For that it is more useful to invert the constitutive equation in order to express the strain-rate $\boldsymbol{D}$ as a function of the deviatoric stress $\boldsymbol{S}$ leading to

$$
\boldsymbol{D}=\frac{1}{2 \eta}\left[1-\frac{\mu p}{\|\boldsymbol{S}\|}\right]_{+} \boldsymbol{S}
$$

with $\eta=\eta_{I}$ or $\eta=\eta_{c}$. The above expressions were introduced by Perzyna ${ }^{45}$ and Duvaut and Lions ${ }^{10}$ and are called the viscoplastic regularization method.

The treatment of the Coulomb friction condition is done using the same type of regularization approach as above (see the work of Ionescu ${ }^{21,22}$ ). A small frictional viscosity $\eta_{f}<<1$ is introduced in the Coulomb friction law (18) and (17) to get

$$
\begin{gathered}
\boldsymbol{u}_{T}=-\frac{1}{\eta_{f}}\left[1-\frac{\mu^{f}\left[-\boldsymbol{\sigma}_{n}\right]_{+}}{\left|\boldsymbol{F}_{b}^{f}\right|}\right]_{+} \boldsymbol{F}_{b}^{f}, \\
\boldsymbol{u}=-\frac{1}{\eta_{f}}\left[1-\frac{\mu_{w}^{f}[p]_{+}}{\left|\boldsymbol{F}_{w}^{f}\right|}\right]_{+} \boldsymbol{F}_{w}^{f} .
\end{gathered}
$$

This formulation of the friction law has the same mathematical structure as the viscoplastic constitutive Equation (A1) and we can use the same iterative decomposition-coordination formulation. In the present case, the regularization frictional viscosity coefficient has been set to $\eta_{f}=0.1 \mathrm{~Pa} \mathrm{~s} \mathrm{~m}^{-1}$.

The treatment of the free-surface and the associated timemoving domain within a Navier-Stokes problem is achieved through an Arbitrary Lagrangian-Eulerian (ALE) method which is rather classical and thus not described here (see, for instance, the work of Hughes et al.,${ }^{20}$ Maury, ${ }^{39}$ Maronnier et al. ${ }^{37}$ and Duarte et $a l .{ }^{9}$ ).

\section{Time discretization}

Let $\Delta t$ be the time step and $\boldsymbol{u}^{k}, \boldsymbol{S}^{k}$, and $p^{k}$ be the values of the unknowns at time $k$. The implicit Euler scheme applied to problem (9) with viscoplastic flow law (A1) gives the following set of nonlinear equations on $\boldsymbol{u}^{k}, \boldsymbol{S}^{k}, p^{k}, \boldsymbol{F}_{b}^{f k}$, and $\boldsymbol{F}_{w}^{f k}$ :

$\rho\left(\frac{\boldsymbol{u}^{k}-\boldsymbol{u}^{k-1}}{\Delta t}+\boldsymbol{u}^{k} \cdot \nabla \boldsymbol{u}^{k}\right)-\operatorname{div} \boldsymbol{S}^{k}+\nabla p^{k}+\boldsymbol{F}_{w}^{f k}=\rho \boldsymbol{g}$ in $\Omega$,

$$
\begin{gathered}
\operatorname{div}\left(\boldsymbol{u}^{k}\right)=0 \quad \text { in } \quad \Omega, \\
\boldsymbol{D}\left(\boldsymbol{u}^{k}\right)=\frac{1}{2 \eta\left(\left\|\boldsymbol{D}\left(\boldsymbol{u}^{k}\right)\right\|, p^{k}\right)}\left[1-\frac{\mu p^{k}}{\left\|\boldsymbol{S}^{k}\right\|}\right]_{+} \boldsymbol{S}^{k}, \\
\boldsymbol{u}^{k}=\frac{-1}{\eta_{f}}\left[1-\frac{\mu_{w}^{f}\left[p^{k}\right]_{+}}{\left|\boldsymbol{F}_{w}^{f k}\right|}\right]_{+} \boldsymbol{F}_{w}^{f k}, \\
\boldsymbol{u}_{T}^{k}=-\frac{1}{\eta_{f}}\left[1-\frac{\mu^{f}\left[-\boldsymbol{\sigma}_{n}^{k}\right]_{+}}{\left|\boldsymbol{F}_{b}^{f k}\right|}\right]_{+} \boldsymbol{F}_{b}^{f k},
\end{gathered}
$$

while the boundary conditions read

$$
\begin{array}{r}
\left(-p^{k} \operatorname{Id}+\boldsymbol{S}^{k}\right) \boldsymbol{n}=0 \quad \text { on } \quad \Gamma_{s}, \\
\boldsymbol{u}^{k} \cdot \boldsymbol{n}=0,\left(-p^{k} \mathrm{Id}+\boldsymbol{S}^{k}\right)_{T}=\boldsymbol{F}_{b}^{f k} \text { on } \Gamma_{b} .
\end{array}
$$

\section{The algorithm at each time step}

Let $r, r_{f}, r_{w}>0$ be the augmented Lagrangian parameters. Let the strain rate multipliers $\dot{\gamma}^{k, n-1}: \Omega \rightarrow \mathbb{R}_{S}^{3 \times 3}$, the slip rate multipliers $\delta^{k, n-1}: \Gamma_{b} \rightarrow \mathbb{R}^{2}$, and the lateral slip rate multiplier $\lambda^{k, n-1}: \Omega \rightarrow \mathbb{R}^{2}$ be known.

Step 1. The first step consists in solving the following linear Stokes-like problem for the velocity field $\boldsymbol{u}^{k, n}$ and the pressure $p^{k, n}$,

$$
\operatorname{div}\left(\boldsymbol{u}^{k, n}\right)=0,
$$

$$
\begin{aligned}
& \rho\left(\frac{\boldsymbol{u}^{k, n}-\boldsymbol{u}^{k-1}}{\Delta t}+\boldsymbol{u}^{k, n-1} \cdot \nabla \boldsymbol{u}^{k, n}\right) \\
& \quad-\operatorname{div}\left(r \boldsymbol{D}\left(\boldsymbol{u}^{k, n}\right)\right)+\nabla p^{k, n}-\frac{2}{w} r_{w} \boldsymbol{u}^{k, n}=\rho \boldsymbol{g} \\
& \quad+\operatorname{div}\left(\boldsymbol{S}^{k, n-1}-r \dot{\gamma}^{k, n-1}\right)+\frac{2}{w}\left(\boldsymbol{F}_{w}^{f k, n-1}-r_{w} \lambda^{k, n-1}\right),
\end{aligned}
$$

with the boundary conditions

$$
\begin{gathered}
\left(r \boldsymbol{D}\left(\boldsymbol{u}^{k, n}\right)-p^{k, n} \mathrm{Id}+\boldsymbol{S}^{k, n-1}-r \dot{\gamma}^{k, n-1}\right) \boldsymbol{n}=0, \quad \text { on } \quad \Gamma_{s}, \\
\boldsymbol{u}^{k, n} \cdot \boldsymbol{n}=0, \quad \text { on } \quad \Gamma_{b}, \\
\left(r \boldsymbol{D}\left(\boldsymbol{u}^{k, n}\right)-p^{k, n} \mathrm{Id}+\boldsymbol{S}^{k, n-1}-r \dot{\gamma}^{k, n-1}\right)_{T} \\
=-r_{f} \boldsymbol{u}_{T}^{k, n}+r_{f} \delta^{k, n-1}+\boldsymbol{F}_{b}^{f k, n-1}, \quad \text { on } \Gamma_{b} .
\end{gathered}
$$


Step 2. First we update the viscosity coefficient $\eta=\eta\left(\left\|\boldsymbol{D}\left(\boldsymbol{u}^{k, n}\right)\right\|, p^{k, n}\right)$ and the yield limit $\kappa=\mu p^{k, n}$. Then, we compute the strain rate multipliers $\dot{\gamma}^{k, n}$ and the slip rate multipliers $\delta^{k, n}$,

$\dot{\gamma}^{k, n}=\frac{1}{2 \eta+r}\left[1-\frac{\mu\left[p^{k, n}\right]_{+}}{\left\|\boldsymbol{S}^{k, n-1}+r \boldsymbol{D}\left(\boldsymbol{u}^{k, n}\right)\right\|}\right]_{+}\left(\boldsymbol{S}^{k, n-1}+r \boldsymbol{D}\left(\boldsymbol{u}^{k, n}\right)\right)$,

$\delta^{k, n}=-\frac{1}{\eta_{f}+r_{f}}\left[1-\frac{\mu^{f}\left[-\sigma_{n}^{k, n-1}\right]_{+}}{\left|\boldsymbol{F}_{b}^{f k, n-1}-r_{f} \boldsymbol{u}_{T}^{k, n}\right|}\right]_{+}\left(\boldsymbol{F}_{b}^{f k, n-1}-r_{f} \boldsymbol{u}_{T}^{k, n}\right)$,

$\lambda^{k, n}=-\frac{1}{\eta_{w}+r_{w}}\left[1-\frac{\mu_{w}^{f}\left[p^{k, n}\right]_{+}}{\left|\boldsymbol{F}_{w}^{f k, n-1}-r_{w} \boldsymbol{u}^{k, n}\right|}\right]_{+}\left(\boldsymbol{F}_{w}^{f k, n-1}-r_{w} \boldsymbol{u}^{k, n}\right)$,

according to the decomposition-coordination formulation coupled with the augmented Lagrangian method.

Step 3. Finally, we update the stress deviator $\boldsymbol{S}^{k, n}$, the tangential stress $\boldsymbol{\sigma}_{T}^{k, n}$, and the lateral stress $\boldsymbol{\sigma}_{\mathbf{Z}}$ using

$$
\begin{gathered}
\boldsymbol{S}^{k, n}=\boldsymbol{S}^{k, n-1}+r\left(\boldsymbol{D}\left(\boldsymbol{u}^{k, n}\right)-\dot{\gamma}^{k, n}\right), \\
\boldsymbol{F}_{b}^{f k, n}=\boldsymbol{F}_{b}^{f k, n-1}-r_{f}\left(\boldsymbol{u}_{T}^{k, n}-\delta^{k, n}\right), \\
\boldsymbol{F}_{w}^{f k, n}=\boldsymbol{F}_{w}^{f k, n-1}-r_{w}\left(\boldsymbol{u}^{k, n}-\lambda^{k, n}\right) .
\end{gathered}
$$

In the present paper, the problem is solved using a finite element formulation. The computational domain $\Omega$ is discretized using triangular finite elements. The finite element spaces for the discretization of $\boldsymbol{u}^{k, n}$ and $p^{k, n}$ are respectively $\mathbb{P}_{2}$ continuous and $\mathbb{P}_{1}$ continuous. The variables $\boldsymbol{S}^{k, n}$ and $\dot{\gamma}^{k, n}$ are discretized using $\mathbb{P}_{1}$ discontinuous finite elements. The variables $\boldsymbol{F}_{b}^{f k, n}$ and $\delta^{k, n}$ on the one hand, and $\boldsymbol{F}_{w}^{f k, n}$ and $\lambda^{k, n}$ on the other hand, are discretized using $\mathbb{P}_{2}$ continuous finite elements. The solution of the Stokes like problem at step 1 is rather standard and we address the reader to the literature for the many techniques available (see, for instance, the work of Pironneau ${ }^{46}$ ). The whole implementation of the solver has been achieved using the software FreeFem.$++{ }^{19}$

\section{APPENDIX B: ANALYTICAL PRESSURE IN THE GRAVITY RELATED FRAME}

Let us consider the topography related frame $(X, Z)$ and the velocity field $(U(X, Z), W(X, Z))^{T}$ in this frame. We denote by $\alpha$ the (constant) slope angle of the topography with respect to the gravity related frame $(x, z)$ and by $(u(x, z), w(x, z))^{T}$ the velocity field in this frame. We have then

$$
\left\{\begin{array}{l}
x=X \cos \alpha+Z \sin \alpha, \\
z=-X \sin \alpha+Z \cos \alpha,
\end{array}\right.
$$

or equivalently

$$
\left\{\begin{array}{l}
X=x \cos \alpha-z \sin \alpha \\
Z=x \sin \alpha+z \cos \alpha .
\end{array}\right.
$$

The analytical pressure in $(X, Z)$ is given by

$$
\begin{aligned}
p(X, Z)= & \rho g\left(\cos \alpha-\sin \alpha \partial_{X} h(X)\right. \\
& \left.-2|\sin \alpha| \frac{\partial_{X} U(X, Z)}{\left|\partial_{Z} U(X, Z)\right|}\right)(h(X)-Z),
\end{aligned}
$$

where $h(X)=Z_{s}$, the height of the free-surface in $(X, Z)$ (i.e., the local thickness of the flow at $X$ ). Indeed, $h(X)-Z$ is the distance between the point $(X, Z)$ and the free surface in the direction of the $Z$ axis. If we denote similarly by $h_{v}(x)=z_{s}$ the vertical height of the free-surface at $x$, it follows from (B2) that

$$
\begin{aligned}
X & =x \cos \alpha-h_{v}(x) \sin \alpha, \\
h(X) & =x \sin \alpha+h_{v}(x) \cos \alpha,
\end{aligned}
$$

where the first line in (B4) indeed relates $x$ and $X$ for a point in the free surface. Differentiating these relations yields

$$
\partial_{X} h(X)=\frac{\sin \alpha+\partial_{x} h_{v}(x) \cos \alpha}{\cos \alpha-\partial_{x} h_{v}(x) \sin \alpha} .
$$

This formula is expressed in terms of the horizontal coordinate $x=X \cos \alpha+h(X) \sin \alpha$ of the point on the free surface. This value is different from the coordinate $x(X, Z)$ defined in (B1). Assume however that $\partial_{x} h_{v}$ is almost constant (meaning that the free surface is almost a straight line), the formula (B5) can be used anyway. With the same assumption, we compute

$$
h(X)-Z=\left(h_{v}(x)-z\right)\left(\cos \alpha+\partial_{X} h \sin \alpha\right) .
$$

For the computation of the velocity derivatives, the change of variable gives

$$
U(X, Z)=u(x, z) \cos \alpha-w(x, z) \sin \alpha,
$$

and using the chain rule,

$$
\begin{aligned}
\partial_{X} U= & \left(\partial_{x} u \cos \alpha-\partial_{z} u \sin \alpha\right) \cos \alpha \\
& -\left(\partial_{x} w \cos \alpha-\partial_{z} w \sin \alpha\right) \sin \alpha, \\
\partial_{Z} U= & \left(\partial_{x} u \sin \alpha+\partial_{z} u \cos \alpha\right) \cos \alpha \\
& -\left(\partial_{x} w \sin \alpha+\partial_{z} w \cos \alpha\right) \sin \alpha .
\end{aligned}
$$

We finally obtain the value of $p(x, z)$ by applying (B3) with $h(X)-Z$ computed by (B6) and dropping terms in $\mathcal{O}\left(\varepsilon^{3}\right)$,

$$
\begin{aligned}
p(x, z)= & \rho g(\cos \alpha \\
& \left.-2|\sin \alpha| \frac{\partial_{X} U(X, Z)}{\left|\partial_{Z} U(X, Z)\right|}\right)\left(h_{v}(x)-z\right) \cos \alpha,
\end{aligned}
$$

with $\partial_{X} U, \partial_{Z} U$ computed by (B8) and (B9), noticing that the terms in $\partial_{X} h(X)$ simplify.

Instead of taking $\alpha$ to be the slope angle of the channel, it is also possible to take for $\alpha$ in (B10) the angle that defines locally a plane parallel to the free surface, that is, $\tan \alpha=-\partial_{x} h_{v}(x)$. Our computations with this local slope angle (not shown) give results very similar to those obtained for constant $\alpha$ shown in Figure 18.

\section{APPENDIX C: ILL-POSEDNESS (LINEAR STABILITY) ANALYSIS FOR THE CONSTANT VISCOSITY DRUCKER-PRAGER FLUID}

We consider the constant viscosity viscoplastic model

$$
\rho\left(\partial_{t} \boldsymbol{u}+\boldsymbol{u} \cdot \nabla \boldsymbol{u}\right)+\nabla p-\operatorname{div} \boldsymbol{S}=\rho \boldsymbol{g},
$$


with $\operatorname{div} \boldsymbol{u}=0$ and

$$
\boldsymbol{S}=\mu_{1} p \frac{\boldsymbol{D}}{\|\boldsymbol{D}\|}+2 \eta_{c} \boldsymbol{D} .
$$

Denoting $\breve{p}=p / \rho$ and $v=\eta_{c} / \rho$, it can be written also as

$$
\partial_{t} \boldsymbol{u}+\boldsymbol{u} \cdot \nabla \boldsymbol{u}=\operatorname{div}\left(\mu_{1} \check{p} \frac{\boldsymbol{D}}{\|\boldsymbol{D}\|}+2 v \boldsymbol{D}\right)-\nabla \check{p}+\boldsymbol{g} .
$$

Then as in Eq. (2.22) in the work of Barker et al., ${ }^{3}$ we can expand the stress terms and get denoting $\boldsymbol{A}=\boldsymbol{D} /\|\boldsymbol{D}\|$,

$$
\begin{aligned}
\partial_{t} \boldsymbol{u}+\boldsymbol{u} \cdot \nabla \boldsymbol{u} & =\left(\frac{\mu_{1} \check{p}}{2\|\boldsymbol{D}\|}+v\right) \Delta \boldsymbol{u}-\frac{\mu_{1} \check{p}}{2\|\boldsymbol{D}\|} \mathcal{A} \partial^{2} \boldsymbol{u} \\
& +\left(\mu_{1} \boldsymbol{A}-\mathrm{Id}\right) \nabla \check{p}+\boldsymbol{g}
\end{aligned}
$$

with $\left(\mathcal{A} \partial^{2} \boldsymbol{u}\right)_{i}=\sum_{j k l} \boldsymbol{A}_{i j} \boldsymbol{A}_{k l} \partial_{j l}^{2} \boldsymbol{u}_{k}$.

Following the arguing of Barker et al. ${ }^{3}$ in order to study the well-posedness, we linearize (C4) around a particular solution $\boldsymbol{u}^{0}, \check{p}^{0}$ and write $\boldsymbol{u} \simeq \boldsymbol{u}^{0}+\widehat{\boldsymbol{u}}, \check{p} \simeq \check{p}^{0}+\widehat{p}$. Considering the high wavenumber limit, we retain only the principal part of the linearized equations on $\widehat{\boldsymbol{u}}, \widehat{p}$, which are $\operatorname{div} \widehat{\boldsymbol{u}}=0$ and

$\partial_{t} \widehat{\boldsymbol{u}}=\left(\frac{\mu_{1} \check{p}}{2\|\boldsymbol{D}\|}+v\right) \Delta \widehat{\boldsymbol{u}}-\frac{\mu_{1} \check{p}}{2\|\boldsymbol{D}\|} \mathcal{A} \partial^{2} \widehat{\boldsymbol{u}}+\left(\mu_{1} \boldsymbol{A}-\mathrm{Id}\right) \nabla \widehat{p}$.

Since we are in the high wavenumber limit, we can consider that the coefficients, related to the base solution, are constant, and we look for normal modes $\widehat{\boldsymbol{u}}=\exp (i \boldsymbol{\xi} \cdot \boldsymbol{x}+\lambda t) \widetilde{\boldsymbol{u}}, \widehat{p}$ $=\exp (i \boldsymbol{\xi} \cdot \boldsymbol{x}+\lambda t) \widetilde{p}$. We get the equations

$$
i \boldsymbol{\xi} \cdot \widetilde{\boldsymbol{u}}=0
$$

$$
\begin{aligned}
\lambda \widetilde{\boldsymbol{u}}= & -\left(\frac{\mu_{1} \check{p}}{2\|\boldsymbol{D}\|}+v\right)|\boldsymbol{\xi}|^{2} \widetilde{\boldsymbol{u}}+\frac{\mu_{1} \check{p}}{2\|\boldsymbol{D}\|} \boldsymbol{A} \boldsymbol{\xi}(\boldsymbol{A} \boldsymbol{\xi} \widetilde{\boldsymbol{u}}) \\
& +i\left(\mu_{1} \boldsymbol{A} \boldsymbol{\xi}-\boldsymbol{\xi}\right) \widetilde{p}
\end{aligned}
$$

Taking the scalar product of the second equation with $\boldsymbol{\xi}$, we get the value of $\widetilde{p}$,

$$
\widetilde{p}=-i \frac{\mu_{1} \check{p}}{2\|\boldsymbol{D}\|} \frac{(\boldsymbol{A} \boldsymbol{\xi} \boldsymbol{\xi})(\boldsymbol{A} \boldsymbol{\xi} \widetilde{\boldsymbol{u}})}{|\boldsymbol{\xi}|^{2}-\mu_{1} \boldsymbol{A} \boldsymbol{\xi} \xi} .
$$

Replacing $\widetilde{p}$ by its value in (C6) yields the eigenvalue problem $\boldsymbol{L} \widetilde{\boldsymbol{u}}=\lambda \widetilde{\boldsymbol{u}}$, with

$$
\begin{aligned}
\boldsymbol{L}= & \frac{\mu_{1} \check{p}}{2\|\boldsymbol{D}\|}\left(\boldsymbol{A} \boldsymbol{\xi}+\frac{\boldsymbol{A} \boldsymbol{\xi} \boldsymbol{\xi}}{|\boldsymbol{\xi}|^{2}-\mu_{1} \boldsymbol{A} \boldsymbol{\xi} \boldsymbol{\xi}}\left(\mu_{1} \boldsymbol{A} \boldsymbol{\xi}-\boldsymbol{\xi}\right)\right)(\boldsymbol{A} \boldsymbol{\xi})^{T} \\
& -\left(\frac{\mu_{1} \check{p}}{2\|\boldsymbol{D}\|}+v\right)|\boldsymbol{\xi}|^{2} \mathrm{Id} .
\end{aligned}
$$

But since $\boldsymbol{\xi} \cdot \widetilde{\boldsymbol{u}}=0$ and we are in two dimensions, $\boldsymbol{\xi}^{\perp}$ is automatically an eigenvector with eigenvalue $\lambda=\xi^{\perp} \cdot L \xi^{\perp} /|\xi|^{2}$, thus

$$
\begin{aligned}
\lambda & =\frac{\mu_{1} \check{p}}{2\|\boldsymbol{D}\|}\left(\boldsymbol{A} \boldsymbol{\xi} \cdot \boldsymbol{\xi}^{\perp}+\frac{\boldsymbol{A} \boldsymbol{\xi} \boldsymbol{\xi}}{|\boldsymbol{\xi}|^{2}-\mu_{1} \boldsymbol{A} \boldsymbol{\xi} \boldsymbol{\xi}} \mu_{1} \boldsymbol{A} \boldsymbol{\xi} \cdot \boldsymbol{\xi}^{\perp}\right) \frac{\boldsymbol{A} \boldsymbol{\xi} \cdot \boldsymbol{\xi}^{\perp}}{|\boldsymbol{\xi}|^{2}}-\left(\frac{\mu_{1} \check{p}}{2\|\boldsymbol{D}\|}+v\right)|\boldsymbol{\xi}|^{2} \\
& =\frac{\mu_{1} \check{p}}{2\|\boldsymbol{D}\|} \frac{\left(\boldsymbol{A} \boldsymbol{\xi} \cdot \boldsymbol{\xi}^{\perp}\right)^{2}+\left(1+v 2\|\boldsymbol{D}\| / \mu_{1} \check{p}\right)\left(\mu_{1}|\boldsymbol{\xi}|^{2} \boldsymbol{A} \boldsymbol{\xi} \boldsymbol{\xi}-|\boldsymbol{\xi}|^{4}\right)}{|\boldsymbol{\xi}|^{2}-\mu_{1} \boldsymbol{A} \boldsymbol{\xi} \boldsymbol{\xi}}
\end{aligned}
$$

If $\lambda>0$ there is ill-posedness since $\lambda$ scales like $|\xi|^{2}$. Since $\boldsymbol{A}^{T}=\boldsymbol{A}, \operatorname{tr} \boldsymbol{A}=0,\|\boldsymbol{A}\|=1$, it follows that $\boldsymbol{A}$ is an orthogonal symmetry. Thus assuming that $\mu_{1}<1$, the denominator of (C9) is positive. Therefore we have to look at the positivity of the numerator or equivalently of

$$
N=q|\boldsymbol{\xi}|^{2} \boldsymbol{A} \boldsymbol{\xi} \boldsymbol{\xi}-|\boldsymbol{\xi}|^{4}+r\left(\boldsymbol{A} \boldsymbol{\xi} \cdot \boldsymbol{\xi}^{\perp}\right)^{2}
$$

with

$$
q=\mu_{1}, \quad r=\frac{1}{1+v \frac{2\|\boldsymbol{D}\|}{\mu_{1} \check{p}}}=\frac{1}{1+\eta_{c} \frac{2\|\boldsymbol{D}\|}{\mu_{1} p}} .
$$

The expression of $N$ in $(\mathrm{C} 10)$ is the same as that in Eq. (2.36) in Ref. 3, except that the values of $q$ and $r$ are different. The arguing of Barker et al. ${ }^{3}$ yields then the condition of ill-posedness (for which $N>0$ ), which is

$$
r>1 / 2 \text { and } q^{2}>4 r(1-r)
$$

In particular, the constant viscosity model is linearly ill-posed for $\mu_{1}$ not too small when $\eta_{c} \frac{2\|\boldsymbol{D}\|}{\mu_{1} p}<1$. Moreover, in the limit $\|\boldsymbol{D}\| \rightarrow 0$, the linearized ill-posedness occurs as soon as $\mu_{1}>0$.
${ }^{1}$ C. Ancey, P. Coussot, and P. Evesque, "A theoretical framework for very concentrated granular suspensions in steady simple shear flow," J. Rheol. 43, 1673-1699 (1999).

${ }^{2}$ B. Andreotti, Y. Forterre, and O. Pouliquen, Les Milieux Granulaires, Entre Fluide et Solide, Savoirs Actuels (EDP Sciences, 2011).

${ }^{3}$ T. Barker, D. G. Schaeffer, P. Bohorquez, and J. M. N. T. Gray, "Well-posed and ill-posed behaviour of the $\mu(I)$-rheology for granular flow," J. Fluid Mech. 779, 794-818 (2015).

${ }^{4}$ E. C. Bingham, Fluidity and Plasticity (Mc Graw-Hill, New York, 1992).

${ }^{5}$ F. Bouchut, E. Fernandez-Nieto, A. Mangeney, and P.-Y. Lagrée, "On new erosion models of Savage-Hutter type for avalanches," Acta Mech. 199(1-4), 181-208 (2008).

${ }^{6}$ F. Bouchut, I. R. Ionescu, and A. Mangeney, "An analytic approach for the evolution of the static/flowing interface in viscoplastic granular flows," Comm. Math. Sci. 14, 2101-2126 (2016).

${ }^{7}$ O. Cazacu and I. R. Ionescu, "Compressible rigid visco-plastic fluids," J. Mech. Phys. Solids 54, 1640-1667 (2006).

${ }^{8}$ G. B. Crosta, S. Imposimato, and D. Roddeman, "Numerical modeling of 2-D granular step collapse on erodible and nonerodible surface," J. Geophys. Res.: Earth Surf. 114, F03020, doi:10.1029/2008JF001186 (2009).

${ }^{9}$ F. Duarte, R. Gormaz, and S. Natesan, "Arbitrary Lagrangian Eulerian method for Navier Stokes equations with moving boundaries," Comput. Methods Appl. Mech. Eng. 193, 4819-4836 (2004).

${ }^{10} \mathrm{G}$. Duvaut and J.-L. Lions, Les Inéquations en Mécanique et en Physique (Dunod, Paris, 1972).

${ }^{11}$ R. Delannay, A. Valance, A. Mangeney, O. Roche, and P. Richard, "Granular and particle-laden flows: From laboratory experiments to fields observations," J. Phys. D: Appl. Phys. (in press).

${ }^{12}$ E. E. Doyle, H. E. Huppert, G. Lube, H. M. Mader, and R. S. J. Sparks, "Static and flowing regions in granular collapses down channels: Insights from a sedimenting shallow water model," Phys. Fluids 19, 106601 (2007). 
${ }^{13}$ M. Farin, A. Mangeney, and O. Roche, "Fundamental changes of granular flow dynamics, deposition, and erosion processes at high slope angles: Insights from laboratory experiments," J. Geophys. Res.: Earth Surf. 119, 504-532, doi:10.1002/2013jf002750 (2014).

${ }^{14}$ M. Fortin and R. Glowinski, Méthodes de Lagrangien Augmenté, Application à la Résolution de Problèmes aux Limites (Dunod, 1982).

${ }^{15}$ P. Favreau, A. Mangeney, A. Lucas, G. Crosta, and F. Bouchut, "Numerical modeling of landquakes," Geophys. Res. Lett. 37, L15305, doi:10.1029/2010GL043512 (2010).

${ }^{16}$ L. Girolami, V. Hergault, G. Vinay, and A. Wachs, "A three-dimensional discrete-grain model for the simulation of dam-break rectangular collapses: Comparison between numerical results and experiments," Granular Matter 14, 381-392 (2012).

${ }^{17}$ R. Glowinski and P. Le Tallec, Augmented Lagrangian and Operator Splitting Method in Non-Linear Mechanics (SIAM Studies in Applied Mathematics, 1989).

${ }^{18}$ J. Hadamard, Sur les Problèmes aux Dérivées Partielles et Leur Signification Physique (Princeton University Bulletin, 1902), pp. 49-52.

${ }^{19}$ F. Hecht, "New development in FreeFem++," J. Numer. Math. 20(3-4), 251-265 (2012).

${ }^{20} \mathrm{~T}$. Hughes, W. Liu, and T. Zimmermann, "Lagrangian-Eulerian finite element formulation for incompressible viscous flows," Comput. Methods Appl. Mech. Eng. 29, 329-349 (1981).

${ }^{21}$ I. R. Ionescu, "Onset and dynamic shallow flow of a viscoplastic fluid on a plane slope," J. Non-Newtonian Fluid Mech. 165(19-20), 1328-1341 (2010).

${ }^{22}$ I. R. Ionescu, "Augmented Lagrangian for shallow viscoplastic flow with topography,” J. Comput. Phys. 242, 544-560 (2013).

${ }^{23}$ I. R. Ionescu, "Viscoplastic shallow flow equations with topography," J. Non-Newtonian Fluid Mech. 193, 116-128 (2013).

${ }^{24}$ I. R. Ionescu, A. Mangeney, F. Bouchut, and O. Roche, "Viscoplastic modeling of granular column collapse with pressure-dependent rheology," J. Non-Newtonian Fluid Mech. 219, 1-18 (2015).

${ }^{25}$ P. Jop, Y. Forterre, and O. Pouliquen, "A constitutive law for dense granular flows," Nature 441, 727-730 (2006).

${ }^{26} \mathrm{P}$. Jop, Y. Forterre, and O. Pouliquen, "Crucial role of sidewalls in granular surface flows: Consequences for the rheology," J. Fluid Mech. 541, 167-192 (2005).

${ }^{27}$ R. R. Kerswell, "Dam break with Coulomb friction: A model for granular slumping,” Phys. Fluids 17, 057101 (2005).

${ }^{28}$ L. Lacaze and R. R. Kerswell, "Axisymmetric granular collapse: A transient 3D flow test of viscoplasticity," Phys. Rev. Lett. 102, 108305 (2009).

${ }^{29}$ L. Lacaze, J. Phillips, and R. R. Kerswell, "Planar collapse of a granular column: Experiments and discrete-element simulations," Phys. Fluids 20, 063302 (2008).

${ }^{30}$ P.-Y. Lagrée, L. Staron, and S. Popinet, "The granular column collapse as a continuum: Validity of a two-dimensional Navier-Stokes model with a $\mu(I)$-rheology," J. Fluid Mech. 686, 378-408 (2011).

${ }^{31}$ E. Lajeunesse, A. Mangeney-Castelnau, and J. P. Vilotte, "Spreading of a granular mass on an horizontal plane," Phys. Fluids 16(7), 2371-2381 (2004).

${ }^{32}$ E. Larrieu, L. Staron, and E. J. Hinch, "Raining into shallow water as a description of the collapse of a column of grains," J. Fluid Mech. 554, 259 (2006).

${ }^{33}$ C. Levy, A. Mangeney, F. Bonilla, C. Hibert, E. Calder, and P. J. Smith, "Friction weakening in granular flows deduced from seismic records at the
Soufrière Hills Volcano, Montserrat,” J. Geophys. Res.: Solid Earth 120, 7536-7557, doi:10.1002/2015jb012151 (2015).

${ }^{34}$ G. Lube, H. E. Huppert, R. S. Sparks, and M. A. Hallworth, "Axisymmetric collapses of granular columns,” J. Fluid Mech. 508, 175 (2004).

${ }^{35}$ A. Mangeney-Castelnau, F. Bouchut, J.-P. Vilotte, E. Lajeunesse, A. Aubertin, and M. Pirulli, "On the use of Saint-Venant equations to simulate the spreading of a granular mass," J. Geophys. Res.: Solid Earth 110, B09103, doi:10.1029/2004JB003161 (2005).

${ }^{36}$ A. Mangeney, O. Roche, O. Hungr, N. Mangold, G. Faccanoni, and A. Lucas, "Erosion and mobility in granular collapse over sloping beds," J. Geophys. Res.: Earth Surf. 115, F03040, doi:10.1029/2009jf001462 (2010).

${ }^{37}$ V. Maronnier, M. Picasso, and J. Rappaz, "Numerical simulation of three dimensional free surface flows," Int. J. Numer. Methods Fluids 42, 697-716 (2003).

${ }^{38}$ N. Martin and J. Monnier, "Inverse rheometry and basal properties inference for pseudoplastic geophysical flows," Eur. J. Mech. - B/Fluids 50, 110-126 (2014).

${ }^{39}$ B. Maury, "Characteristics ALE method for the unsteady 3D Navier-Stokes equations with a free surface,” Int. J. Comput. Fluid Dyn. 6, 175-188 (1996).

${ }^{40}$ C. Meruane, A. Tamburrino, and O. Roche, "On the role of the ambient fluid on gravitational granular flow dynamics," J. Fluid Mech. 648, 381 (2010).

${ }^{41}$ GDR MiDi Group, “On dense granular flows,” Eur. Phys. J. E 14, 341-365 (2004).

${ }^{42}$ L. Moretti, A. Mangeney, Y. Capdeville, E. Stutzmann, C. Christian Huggel, D. Schneider, and F. Bouchut, "Numerical modeling of the Mount Steller landslide flow history and of the generated long period seismic waves," Geophys. Res. Lett. 39, L16402, doi:10.1029/2012GL052511 (2012).

${ }^{43}$ L. Moretti, K. Allstadt, A. Mangeney, Y. Capdeville, E. Stutzmann, and F. Bouchut, "Numerical modeling of the Mount Meager landslide constrained by its force history derived from seismic data," J. Geophys. Res.: Solid Earth 120(4), 2579-2599, doi:10.1002/2014jb011426 (2015).

${ }^{44}$ T. C. Papanastasiou, "Flow of materials with yield," J. Rheol. 31, 385-404 (1987).

${ }^{45}$ P. Perzyna, "Fundamental problems in viscoplasticity," Adv. Appl. Mech. 9, 243-377 (1996).

${ }^{46}$ O. Pironneau, Finite Element Methods for Fluids (John Wiley \& Sons Ltd., Chichester, 1989).

${ }^{47}$ S. B. Savage, "The mechanics of rapid granular flows," Adv. Appl. Mech 24, 289-366 (1984).

${ }^{48}$ L. E. Silbert et al., "Granular flow down an inclined plane: Bagnold scaling and rheology," Phys. Rev. E 64, 051302 (2001).

${ }^{49}$ D. Schaeffer, "Instability in the evolution-equations describing incompressible granular flow,” J. Differ. Equations 66(1), 19-50 (1987).

${ }^{50}$ D. G. Schaeffer and E. B. Pitman, "Ill-posedness in three-dimensional plastic flow," Commun. Pure Appl. Math. 41(7), 879-890 (1988).

${ }^{51}$ L. Staron and E. J. Hinch, "Study of the collapse of granular columns using 2D discrete-grains simulation," J. Fluid Mech. 545, 1 (2005).

${ }^{52}$ N. Taberlet, P. Richard, A. Valance, R. Delannay, W. Losert, J. M. Pasini, and J. T. Jenkins, "Super stable granular heap in thin channel," Phys. Rev. Lett. 91, 264301 (2003).

${ }^{53}$ M. Yamada, A. Mangeney, Y. Matsushi, and L. Moretti, "Estimation of dynamic friction of the Akatani landslide from seismic waveform inversion and numerical simulation," Geophys. J. Int. 206(3), 1479-1486 (2016).

${ }^{54}$ R. Zenit, "Computer simulations of the collapse of a granular column," Phys. Fluids 17, 031703 (2005). 